\title{
Cultural Influences on Neural Basis of Inhibitory Control
}

Narun Pornpattananangkul ${ }^{1,2}$, Ahmad R. Hariri ${ }^{3}$, Tokiko Harada ${ }^{1,4}$, Yoko Mano ${ }^{1,5}$, Hidetsugu Komeda $^{1,5}$, Todd B. Parrish ${ }^{6}$, Norihiro Sadato ${ }^{4}$, Tetsuya Iidaka ${ }^{7}$, Joan Y. Chiao ${ }^{1,8}$

${ }^{1}$ Department of Psychology and Interdepartmental Neuroscience Program, Northwestern University, Evanston, IL; ${ }^{2}$ Department of Psychology, National University of Singapore, Singapore; ${ }^{3}$ Department of Psychology \& Neuroscience and Institute for Genome Sciences \& Policy, Duke University, Durham, NC; ${ }^{4}$ National Institute for Physiological Sciences, Okazaki, Japan; ${ }^{5}$ Department of Psychology, Kyoto University; ${ }^{6}$ Department of Radiology, Northwestern University, Evanston, IL; ${ }^{7}$ Department of Psychiatry, Nagoya University, Nagoya, Japan; ${ }^{8}$ International Cultural Neuroscience Consortium, Highland Park, IL

Please address correspondences to:

Narun Pornpattananangkul

Department of Psychology

National University of Singapore

28 Medical Drive \#04-19

Singapore 117456

Email: psypn@nus.edu.sg and nonnarun@u.northwestern.edu

and

Joan Y. Chiao

International Cultural Neuroscience Consortium

1954 First St. \#355

Highland Park, IL 60035

Email: joan.chiao@gmail.com 


\title{
Running Head: CULTURE AND NEURAL BASIS OF INHIBITORY CONTROL
}

\author{
Abstract \\ Research on neural basis of inhibitory control has been extensively conducted in various \\ parts of the world. It is often implicitly assumed that neural basis of inhibitory control is \\ universally similar across cultures. Here, we investigated the extent to which culture modulated \\ inhibitory-control brain activity at both cultural-group and cultural-value levels of analysis. \\ During fMRI scanning, participants from different cultural groups (including Caucasian- \\ Americans and Japanese-Americans living in the United States and native Japanese living in \\ Japan) performed a Go/No-Go task. They also completed behavioral surveys assessing cultural \\ values of behavioral consistency, or the extent to which one's behaviors in daily life are \\ consistent across situations. Across participants, the Go/No-Go task elicited stronger neural \\ activity in several inhibitory-control areas, such as the inferior frontal gyrus (IFG) and anterior \\ cingulate cortex (ACC). Importantly, at the cultural-group level, we found variation in left IFG \\ (L-IFG) activity that was explained by a cultural region where participants lived in (as opposed to \\ race). Specifically, L-IFG activity was stronger for native Japanese compared to Caucasian- and \\ Japanese-Americans, while there was no systematic difference in L-IFG activity between \\ Japanese- and Caucasian-Americans. At the cultural-value level, we found that participants who \\ valued being "themselves" across situations (i.e., having high endorsement of behavioral \\ consistency) elicited stronger rostral ACC activity during the Go/No-Go task. Altogether, our \\ findings provide novel insight into how culture modulates the neural basis of inhibitory control. \\ Key words: cultural neuroscience, inhibitory control, action-monitoring, inferior frontal gyrus, \\ anterior cingulate cortex, behavioral consistency
}




\section{Running Head: CULTURE AND NEURAL BASIS OF INHIBITORY CONTROL}

\section{Introduction}

In recent decades, cognitive neuroscientists have extensively investigated the neural basis of inhibitory control with functional neuroimaging (Criaud \& Boulinguez, 2013). In one of the most commonly used tasks, the Go/No-Go, participants are asked to respond to targets, but inhibit their response to non-targets. The Go/No-Go task has been employed in more than 45 neuroimaging studies as well as in several meta-analyses (e.g., Buchsbaum, Greer, Chang, \& Berman, 2005; Criaud \& Boulinguez, 2013; Simmonds, Pekar, \& Mostofsky, 2008; Swick, Ashley, \& Turken, 2011). Although several of these studies have been conducted in non-Western countries, especially in Japan (e.g., Chikazoe, Konishi, Asari, Jimura, \& Miyashita, 2007; Hirose et al., 2012; Nakata, Inui, Wasaka, Akatsuka, \& Kakigi, 2005; Watanabe et al., 2002; Zheng, Oka, Bokura, \& Yamaguchi, 2008), a systematic, cultural comparison of the inhibitory-control brain activity has not yet been conducted.

On the other hand, research in cultural psychology suggests that individualistic and collectivistic cultures differ in inhibitory control (Matsumoto, Yoo, Hirayama, \& Petrova, 2005; Oh \& Lewis, 2008). Such cultural research has been conducted at two levels of analyses, namely (1) the cross-cultural level (cultural-group), or as a comparison between cultural groups that are associated with individualistic (e.g., Westerners) and collectivistic (e.g., East Asians) cultures (Markus \& Kitayama, 1992; Nisbett, Peng, Choi, Norenzayan, 2001) as well as (2) the withinculture level (cultural-value), or as an investigation of within-group variation of values comprising the cultural dimension of individualism and collectivism (Na et al., 2010). Behavioral consistency, or how consistent people behave across situations (Funder \& Colvin, 1991; Suh, 2002), is an example of an individualistic cultural value that may be related to inhibitory control.

First, for the cultural-group level of analysis, prior cross-cultural behavioral studies of culture and inhibitory control have argued that growing up in a place with a strong collectivistic culture (such as, East Asian countries) where self-control is valued through strict teaching and parenting styles seems to necessitate and reinforce inhibitory control (Chao \& Tseng, 2002; Kwon, 


\section{Running Head: CULTURE AND NEURAL BASIS OF INHIBITORY CONTROL}

2002; Wang \& Mao, 1996). Empirical evidence for cultural-group differentiation in inhibitory control mainly comes from early childhood research. For instance, three to four year-old children in East Asian countries are often found to outperform U.S. children in behavioral measures of inhibitory control, such as the Stroop task (Oh \& Lewis, 2008; Sabbagh, Xu, Carlson, Moses, \& Lee, 2006). In a more recent developmental event-related potential (ERP) study, researchers investigated differences in inhibitory control between Chinese-Canadian and Caucasian-Canadian 5-year-old children using a Go-NoGo task (Lahat, Todd, Mahy, Lau, \& Zelazo, 2010). Most of these Chinese-Canadian participants spoke language other than English at home, suggesting a strong cultural tie to their Chinese identity. While there were no cultural differences in behavioral performance between the two groups of children, Chinese-Canadian children elicited stronger amplitude of the N2, an inhibitory-control ERP component that was localized at the inferior frontal (ventrolateral) gyrus in this study. This suggests that cultural differences in inhibitory-control brain activity occurs during early childhood, even in the absence of the differences in behavioral performance.

As for the cultural-value level of analysis, inhibitory control may also be associated with a cultural value that is related to the individualistic-collectivistic dimension. One way that cultures vary is in behavioral consistency, which suggests that cultures differ in how consistently individuals behave across contexts (Funder \& Colvin, 1991; Suh, 2002). Collectivists often value being flexible and behaving according to social contexts, whereas individualists value being "themselves" across contexts and behaving consistently across situations (Markus \& Kitayama, 1991). For instance, when asked to describe themselves, Japanese varied their self-descriptions across situations (e.g., alone, with peers, or with a teacher) more than Americans did (Kanagawa, Cross, \& Markus, 2001). Moreover, Americans, but not Koreans, attribute greater psychological well-being, social skills and social desirability to peers who display greater behavioral consistency (Suh, 2002). Additionally, recently Hardin and colleagues (Hardin, 2006; Hardin, Leong, \& Bhagwat, 2004) have factor analyzed one of the most commonly used self-report scales for measuring individualistic- 


\section{Running Head: CULTURE AND NEURAL BASIS OF INHIBITORY CONTROL}

collectivistic values, called the self-construal scale (SCS) (Singelis, 1994). One factor, or a group of scale items, that was emerged from this factor-analysis method is behavioral consistency. Hence, behavioral consistency is one of the building blocks of individualistic-collectivistic values, and its contribution is independent from other factors within the individualistic-collectivistic dimension. Similar to previous research that analyzed self-descriptions across situations (e.g., Kanagawa et al., 2001), these behavioral consistency items from Hardin's and colleague studies $(2006 ; 2004)$ represent items that ask how consistent individuals behaves (1) across environments they are in and (2) across people who they are with.

Importantly, valuing high behavioral consistency among individualists may provide limited opportunities for people to practice their inhibitory control. For instance, in everyday life, people with high behavioral consistency may be less likely to switch their cognitive sets from one situation to another, or to inhibit their behaviors according to changes in an environment. This reasoning is in line with research on cultural acculturation. Particularly, low level of cultural acculturation among immigrants (or less integration with the host culture) corresponds to an immigrant's relatively poorer performance on inhibitory control tasks, such as the Wisconsin Card Sorting Test (WCST; Grant \& Berg, 1948) and the Stroop test (Coffey, Marmol, Schock, \& Adams, 2005; Razani, Burciaga, Madore, \& Wong, 2007). Although the process of acculturation is not identical to behavioral consistency, acculturation research provides a reason to speculate that not having a chance to shift one's mindset across different environments (e.g., high in behavioral consistency or low in cultural acculturation level) may lead to a poorer inhibitory-control ability.

Taken together, previous behavioral research has indicated a relationship between individualistic-collectivistic culture and inhibitory control, at both cultural-group (i.e., across cultures, such as East Asians vs. Westerners) and cultural-value (i.e., within-culture such as people with different level of behavioral-consistency) levels. Nonetheless, how culture, as defined by these two levels of analysis, influences the neurobiological basis of inhibitory control remains largely unknown. 


\section{Running Head: CULTURE AND NEURAL BASIS OF INHIBITORY CONTROL}

Investigating cultural influences on the neurobiological basis of inhibitory control necessitates adding another analytic layer to cultural differentiation that would not otherwise be shown in behavior. Cultural differentiation in neural responses can occur in the absence of cultural differentiation in behavioral responses (Chiao et al., 2009a). For instance, as mentioned earlier, a developmental Go/No-Go ERP study with 5-year-old children showed cultural differentiation in inhibitory-control at the neural level, but not at the behavioral level (Lahat et al., 2010). Equally important, studying neural activity related to both cultural-group and cultural-value levels of analysis may also reveal how different aspects of culture manifest in the brain. While early research in cultural neuroscience has demonstrated neural variation between cultural groups from different countries (for a review, see Han et al., 2013), we have previously demonstrated that, irrespective of whether the participants are Caucasian Americans living in the United States or Native Japanese living in Japan, cultural values of individualism-collectivism modulate neural responses in a selfjudgment task (Chiao et al., 2009b). In fact, such neural variation as a function of cultural values within and across cultural groups has been found in other studies (e.g., de Greck et al., 2012; Sul, Choi, \& Kang, 2011). Hence, cultural influences on brain function may be observed in both cultural-group and cultural-value levels of analysis (Chiao et al., 2010; Han et al., 2013). Accordingly, it is possible that cultural groups and cultural values may modulate different aspects of neurobiological mechanisms underlying inhibitory control processes.

A distributed cortical network, or the inhibitory-control network, is typically recruited during the Go-NoGo task. Two prominent inhibitory-control areas are the inferior frontal (ventrolateral) gyrus (IFG) and anterior cingulate cortex (ACC). For instance, higher activity in the IFG associates with successful inhibition of irrelevant responses (Swick et al., 2011). Reduction in the IFG activity is associated with deficit in response inhibition in cases of sleep deprivation (Chuah, Venkatraman, Dinges, \& Chee, 2006) and manic disorder (Mazzola-Pomietto, Kaladjian, Azorin, Anton, \& Jeanningros, 2009). While traditionally viewed as lateralized to the right hemisphere (de Zubicaray, Andrew, Zelaya, Williams, \& Dumanoir, 2000; Garavan, Ross, \& Stein, 1999), recently 


\section{Running Head: CULTURE AND NEURAL BASIS OF INHIBITORY CONTROL}

fMRI and lesion studies have begun to demonstrate the critical role of the left IFG (L-IFG) for performing efficiently and successfully in the Go/No-Go task (Hirose et al., 2012; Swick, Ashley, \& Turken, 2008; Swick et al., 2011; Tamm, Menon, \& Reiss, 2002).

As for the ACC, greater activation in the ACC during the task has been found among participants who have strong error and conflict monitoring tendencies, including patients with obsessive-compulsive disorder (OCD) (Maltby, Tolin, Worhunsky, O'Keefe, \& Kiehl, 2005) and major depression disorder (MDD) (Langenecker et al., 2007). Within the ACC, the rostral portion (rACC) seems to play a role in error processing (e.g., conflicts occurring when making errors), whereas the dorsal portion (dACC) is associated with monitoring conflicts (e.g. seeing non-target) (Fassbender et al., 2004). Accordingly, the current study employed the Go/NoGo task to elicit inhibitory-control processes, and focused on the IFG and ACC areas that are reliably elicited by this task, as well as other inhibitory-control tasks (e.g., Buchsbaum et al., 2005; Criaud \& Boulinguez, 2013; Simmonds et al., 2008; Swick et al., 2011).

Simply comparing between people who live in the collectivistic and individualistic countries may not be able to rule out whether the observed cultural-group differentiation is due to cultural influences or, alternatively, genetic variations between people of different races living in different countries. This is especially important given that (1) the observed cultural-group differentiation in inhibitory control between East Asians and U.S. people started quite early in life (Oh \& Lewis, 2008; Sabbagh et al., 2006), (2) recent twin studies have demonstrated that both genetic and environmental factors account for individual differences in inhibitory control (Gagne \& Saudino, 2010; Gagne, Saudino, \& Asherson, 2011), and (3) there have been cases in which genetic differences explain variation in behaviors of people from different countries (Chiao \& Blizinsky, 2010; Mrazek, Chiao, Blizinsky, Lun, \& Gelfand, 2013). To examine the possible genetic effect due to racial differences when conducting cross-cultural experiments, one may add another "control" group in addition to the two cultural groups who live in different geographical regions. People in this control group should grow up in the same cultural region with another group, 


\section{Running Head: CULTURE AND NEURAL BASIS OF INHIBITORY CONTROL}

but are of the same race with the other group.

The aim of the present study is to examine how culture influences the neurobiological basis of inhibitory control at both cultural-group and cultural-value levels of analysis. To examine cultural variation in inhibitory-control brain activity at the cultural-group level, here we compared three groups of people living in two distinct cultures: Caucasian-Americans and JapaneseAmericans who lived in the U.S and Native Japanese who lived in Japan. Japanese-Americans were included as a control group to control for racial differences between Caucasian Americans and Native Japanese. That is, if variation in inhibitory-control neural responses is explained by culture where one lives in, we hypothesize that variation in inhibitory-control neural responses would be observable between people living in Japan and those living in the US, regardless of whether they are Caucasian Americans or Japanese Americans. Alternatively, if variation in inhibitory-control neural responses is explained by racial differences, then we hypothesize that Caucasian Americans would exhibit different inhibitory-control neural responses from people of Japanese descent, regardless of whether they have grown up in the US (i.e., Japanese Americans) or in Japan (i.e., Native Japanese). To examine how cultural values affect inhibitory-control brain activity, we investigated the relationship between behavioral consistency and the neural basis of inhibitorycontrol. We specifically tested whether cultural groups and cultural value of behavioral consistency modulate two main inhibitory-control areas: the IFG (Chuah et al., 2006; Swick et al., 2011), and the ACC (Fassbender et al., 2004).

\section{Methods}

\subsection{Participants}

Sixty-five right-handed, paid volunteers with normal or corrected-to-normal vision participated in this study. Seven participants were excluded due to a) technical problems during the scanning session (3), b) excessive head movement in which their movement parameter exceeded our a priori cutoff of $5 \mathrm{~mm}(2)$, and c) poor behavioral performance (i.e., having hit rate less than $4 \mathrm{SD}$ from the mean) in the Go/No-Go blocks (2). Thus, we analyzed data from fifty-eight 


\section{Running Head: CULTURE AND NEURAL BASIS OF INHIBITORY CONTROL}

participants including 20 Caucasian Americans living in the United States (14 females, $M=24.35$ years, $S D=6.1)$, 16 Japanese Americans living in the United States ( 6 females, $M=23.43$ years, $S D=3.2)$ and 22 Native Japanese living in Japan ( 9 females, $M=21.96$ years, $S D=4.3$ ). We recruited Japanese Americans who self-reported as the first or second generation Japanese Americans, with Native Japanese parents, born and raised in the US or moved to the US by childhood. Furthermore, they all were native English speakers and couldn't read and write Japanese except for only one participant who could speak Japanese a little. ${ }^{1}$ All volunteers provided informed consent prior to participation. This study was approved by the Ethics Committees of Northwestern University, Nagoya University and the National Institute for Physiological Sciences.

\subsection{Procedure}

All participants completed a battery of fMRI tasks in a fixed order, including a emotionprocessing task (Hariri, Tessitore, Mattay, Fera, \& Weinberger, 2002), Go/No-Go task, and selfreference task (Chiao et al., 2009b). This paper examines data from the Go/No-Go task only. Other data from the experimental battery have been reported elsewhere, e.g., the self-reference task (Chiao et al., 2009b) or are currently in a publication process, e.g., the emotion-processing task. A block-design version of the Go/No-Go task used previously (Brown, Manuck, Flory, \& Hariri, 2006; Horn, Dolan, Elliott, Deakin, \& Woodruff, 2003) was adapted for the cross-cultural fMRI paradigm (Figure 1). Participants were asked to respond as quickly and as accurately as possible

\footnotetext{
${ }^{1}$ We employed Japanese-American participants as a control group. They grew up in the same cultural region with Caucasian-Americans participants, but are of the same racial and ethnic origin as Native Japanese participants. The fact that all but one of Japanese-American participants cannot understand Japanese may reflect that they are culturally closer to Caucasian-Americans than Native Japanese. This approach is different from some previous research. In an aforementioned Go/No-Go ERP study (Lahat et al., 2010), for instance, Chinese-Canadian 5year-old children were compared to Caucasian-Canadian 5-year-old children in terms of their ERPs. Eighty-eight percent of the Chinese-Canadian participants in this ERP study spoke language other than English at home. Accordingly, Chinese-Canadian participants in this study may be culturally closer to Native Chinese. Hence, the differences between the two groups of participants in Lahat and colleagues' (2010) study may be more appropriately attributed to cultural effects than the differences between Japanese American and Caucasian American participants in the current study.
} 


\section{Running Head: CULTURE AND NEURAL BASIS OF INHIBITORY CONTROL}

with their right index finger to every letter (hereby "target") except for the designated "nontarget." English letters were used for Caucasian American and Japanese American groups with "v" as a nontarget, while Japanese Katakana letters were used for Native Japanese group with “レ” (RE) as a nontarget. These two non-target letters were similar in both geometry and the frequency of their usage. Specifically, the letter "v" is used around 1\% among English letters (Lewand, 2000; http://www.oxforddictionaries.com), while the letter “レ” is used 1.7\% among Japanese Katakana letters (Chikamatsu, Yokoyama, Nozaki, Long, \& Fukuda, 2000). The target letters were drawn from the same number of letters across both English and Japanese versions. There were two Block conditions: Block A, the Go condition, where 20 targets were presented, and Block B, the Go/NoGo condition, where 10 targets and 10 nontargets were presented. Each of 20 letters in each block was presented for $500 \mathrm{~ms}$, followed by an inter-stimulus interval of $1 \mathrm{~s}$, giving rise to a 30 -s block. Blocks were separated by 20 -s rest periods, where a fixation cross was shown. The order of blocks was pseudo-randomized as ABBABA, giving a total of 120 stimuli presentations during this 8minute task. There was no signal of any kind to indicate which block condition participants were currently in. Moreover, participants were not told about the block structure of the task nor about the order of the blocks. Participants practiced for 2 minutes in the scanner prior to the actual task.

Behavioral performance indices in the task included reaction times and accuracy. Both indices were used given a potential speed-accuracy tradeoff characteristic of a Go/NoGo task (Bogacz, Wagenmakers, Forstmann, \& Nieuwenhuis, 2010; Perri, Berchicci, Spinelli, \& Di Russo, 2014). Reaction times were in milliseconds (ms) and based on an average value from all individual trials. To separate reaction times from accuracy indices, reaction times from all trials in which the designated key was pressed (regardless of accuracy) were analyzed. As for accuracy, accuracy rates were in percentage of correct responses (i.e., pressing to targets and not pressing for nontargets). After scanning, participants completed a battery of questionnaires. 
Running Head: CULTURE AND NEURAL BASIS OF INHIBITORY CONTROL

Cultural values in behavioral consistency were measured through items in the SelfConstrual Scale (SCS) (Singelis, 1994). Based on previous exploratory factor analyses (Hardin, 2006; Hardin et al., 2004)2, two items were identified: 1) "I act the same way no matter who I am with" and 2) "I am the same person at home that I am at school." Participants were asked in their native language if they endorsed each of these items. 7-point Likert-type items were used $(1=$ strongly disagree and $7=$ strongly agree). Scores for these items were then averaged. Higher scores reflected a higher behavioral consistency tendency. Cronbach's Alpha of behavioral consistency items in the current study (.57) was almost identical to that in the previous study with a sample size of 340 subjects (.59) (Hardin, 2006), indicating similar levels of internal consistency across studies. This relatively low alpha value is expected given the small number of items (2) (Cortina, 1993; Nunnally, 1978). High consistency between the two items also reflects in the fact that the first item of behavioral consistency (see item 1) above) correlated with the second item (see item 2$)$ above) at the numerically highest magnitude $(r(56)=.40, p=.002)$ compared to its correlational magnitude with other items in the SCS scale (range: $.01-.33, M$ $=.31, S D=.10)$. Moreover, to compare Cronbach's alpha among groups of participants, we employed the Cocron R-package (Diedenhofen \& Musch, 2014). We found no significant difference in Cronbach's alpha among the groups $\left(\chi^{2}(2)=0.43, p=.81\right)$.

\subsection{Imaging parameters}

\footnotetext{
${ }^{2}$ The SCS (Singelis, 1994) has been traditionally viewed as composing of two broad factors: interdependent and independent self-construal styles. These two factors were emerged from Singelis's original principle component analysis. Recent evidence, however, has questioned this two-factor structure of the SCS scale (Cross, Hardin, \& Gercek-Swing, 2011; Hardin, 2006; Hardin et al., 2004; Levine et al., 2003). Hardin and colleague (2004; 2006), in particular, suggest a multidimensional structure of the scale. In multiple experiments (Hardin, 2006; Hardin et al., $2004)$, they used exploratory factor analyses on larger groups of participants $(\mathrm{N}>4,000)$ and reliably found six factors. Behavioral consistency, consisting of two items, has consistently emerged as one of these factors. These items were originally included in the independent factor of the SCS scale. Recent cultural neuroscience researchers have been encouraged to adopt the multidimensional structure of the SCS (Ma et al., 2014), as this approach allows researchers to focus on each specific cultural-value factor. In fact, we have previously reported the usefulness of this approach in our recent fMRI on neural correlates of emotional pain perception (Cheon et al., 2013).
} 


\section{Running Head: CULTURE AND NEURAL BASIS OF INHIBITORY CONTROL}

Functional brain images were acquired at two facilities, the Center for Advanced Magnetic Resonance Imaging (CAMRI) facility located in the Northwestern Medical Hospital in Chicago, IL, USA, and the National Institute for Physiological Sciences (NIPS) in Okazaki, Japan. Scanning in the US occurred on a comparable 3.0-Tesla Siemens Trio MRI scanner and scanning in Japan occurred on a 3.0-Tesla Siemens Allegra MRI scanner. We acquired functional images by using T2*-weighted, gradient echo, echo planar imaging sequences [repetition time $(\mathrm{TR})=2,000 \mathrm{~ms}$; echo time $(\mathrm{TE})=25 \mathrm{~ms}$; flip angle $=70^{\circ} ; \mathrm{FOV}=20 \mathrm{~cm}, 64 \times 64$ matrix; 34 slices; voxel size $=3.0$ $\left.\times 3.0 \times 3.0 \mathrm{~mm}^{3}\right]$. A high-resolution anatomical T1-weighted image was also acquired $[\mathrm{TR}=2,300$ $\mathrm{ms} ; \mathrm{TE}=2.91 \mathrm{~ms} ;$ flip angle $=9^{\circ} ; \mathrm{FOV}=256 \mathrm{~mm} ; 256 \times 256$ matrix; 176 slices; voxel size $=1.0$ $\left.\times 1.0 \times 1.0 \mathrm{~mm}^{3}\right]$ for each subject. All stimuli were presented using Presentation software (Neurobehavioral Systems, Albany, CA) and projected onto a half-transparent viewing screen located behind the head coil. Subjects viewed the projected stimuli through a mirror.

Because the data was collected at two fMRI sites (i.e., the US and Japan), it is important to consider possible confounding variation in neural activation patterns based solely on site differences. Past studies have demonstrated that under some circumstances, data from different fMRI scanners are comparable, and offered several methods to minimize and measure between-site influence on fMRI data (Friedman \& Glover, 2006; Sutton et al., 2008). We previously described in detail on how we employed these methods and accounted for possible confounding, sitedependent effects in fMRI between these sites (Chiao et al., 2009b). Briefly, we first utilized nearly identical fMRI vendor's instrumentation and imaging parameters that resulted in negligible interscanner variability (Friedman \& Glover, 2006). Second, we conducted an inter-scanner reliability test by comparing signal-to-noise ratio (SNR) (Parrish, Gitelman, LaBar, \& Mesulam, 2000) of the data from four participants ( 1 female, 3 males, $M=33.8$ years, $S D=9.7$ years) collected at the two facilities. This test was done around the same time with the experiment. We did not find any suggestion of the confounding site-dependent effects (for details, see Chiao et al., 2009b). Results 


\section{Running Head: CULTURE AND NEURAL BASIS OF INHIBITORY CONTROL}

indicated no significant difference in SNR $(t(3)=21.04, p=0.18)$ across scanner sites. We conclude that it is unlikely that variation in scanner site performance can explain any differences in neural activation observed between the two sites. Additionally, as mentioned below, this study employed the subtraction paradigm in a block design by comparing contrasts based upon differences in brain activity across blocks. This subtraction method would help control brain activity differences based on stimulus displays between sites.

\subsection{Imaging processing and statistical analysis}

\subsubsection{Imaging pre-processing}

Functional images were analyzed using SPM8 software (Wellcome Department of Imaging Neuroscience, London, UK) implemented in Matlab (Mathworks, Cherborn, MA). The first six volumes were discarded due to unsteady magnetization. All of the remaining volumes were realigned spatially to the first volume and a mean image was created. After a high-resolution image was coregistered onto the mean image, all volumes were normalized to the Montreal Neurological Institute (MNI) space using a transformation matrix obtained from the normalization process of the high-resolution image of each individual subject to the MNI template. The normalized images were then spatially smoothed with an 8-mm Gaussian kernel.

\subsubsection{Whole-brain analyses}

After preprocessing, whole-brain statistical analyses for each individual subject were conducted using the general linear model (Friston et al., 1994). At the first level, each block of trials was modeled by convolving with a hemodynamic response function. A linear regressor was also applied as a regressor of no-interest to filter out low-frequency drift. In the subtraction analysis, two task conditions based on blocks [Go and Go/No-Go] were modeled separately. Random effects analyses were conducted by taking the individual $[\mathrm{Go} / \mathrm{No}-\mathrm{Go}>\mathrm{Go}$ ] contrast images that 


\section{Running Head: CULTURE AND NEURAL BASIS OF INHIBITORY CONTROL}

represented neural responses underlying inhibitory control to the second level for statistical analyses.

For the whole-brain random effects analyses, we employed the 3dClustSim method, implemented in AFNI version 16.1 .04 (http://afni.nimh.nih.gov/; Cox, 1996), to control for multiple statistical testing. Based on Monte Carlo simulations with second-nearest neighbor clustering and 2-sided thresholding, we set the height threshold at $p<0.005$ and the spatial extent threshold at $\mathrm{k}>40$ voxels. This corresponds to an empirically driven clusterwise threshold of $p<$ 0.05 across the whole brain, accounted for spatial correlations between BOLD signal changes in neighboring voxels.

First, to overview whole-brain activation patterns of inhibitory control across participants, a whole-brain voxel-wise one-sample analysis with $[\mathrm{Go} / \mathrm{No}-\mathrm{Go}>\mathrm{Go}$ ] contrast was performed (Table 2, Supplementary Figure 1). Second, to investigate variation in inhibitory-control brain activity at the cultural-group level, we conducted whole-brain analyses using one-way ANOVA with the $[\mathrm{Go} / \mathrm{No}-\mathrm{Go}>\mathrm{Go}$ ] contrast, comparing cultural groups [Caucasian Americans, Japanese Americans, and Native Japanese]. Specifically in this one-way ANOVA model, subjects were treated as random effects in the second level. For the design specification of our one-way ANOVA model, we used the default settings in SPM8. For instance, the measurements between cultural groups were assumed to be independent, and the measurements in each cultural group were allowed to have unequal variance. We also did not specify grand mean scaling, and ANCOVA. These settings resulted in a model, consisting of three regressors (one for each cultural group) without an intercept. Given our primary emphasis on investigating the culture-driven and race-driven hypotheses, we focused our analyses on two sets of planed contrasts. Specifically, to test the culture-driven hypothesis (i.e., whether a culture where individuals live in influenced their inhibitory-control brain activity), the first set of contrasts included [Native Japanese $>$ (Caucasian Americans \& Japanese Americans)] and [(Caucasian Americans \& Japanese Americans) $>$ Native 


\section{Running Head: CULTURE AND NEURAL BASIS OF INHIBITORY CONTROL}

Japanese]. To test the race-driven hypothesis (i.e., whether races, as opposed to cultures, influenced inhibitory-control brain activity), the second set of contrasts included [Caucasian Americans > (Japanese Americans \& Native Japanese)] and [(Japanese Americans \& Native Japanese) > Caucasian Americans] (Table 3). Third, to investigate cultural variation in inhibitory-control brain activity, a whole-brain multiple regression analysis was performed with the [Go/No-Go $>$ Go] contrast with behavioral consistency as a covariate (Table 3). Similar to the one-way ANOVA model, subjects were treated as random effects in this second-level regression model. For the design specification of our regression model, we used the default settings in SPM8. These settings resulted in a model with an intercept and behavioral consistency as a covariate of interest. MNI coordinates were converted to Talairach using a nonlinear transformation (http://imaging.mrccbu.cam.ac.uk/imaging/MniTalairach). Brodmann areas and brain regions were identified based on the Talairach Atlas (Talairach \& Tournoux, 1988).

\subsubsection{A-priori regions of interest analyses}

In addition to the whole-brain analyses, based on our a priori hypotheses, region of interest analyses (ROIs) were conducted on two hypothesized regions: the IFG and the ACC. The ROIs were defined using $6 \mathrm{~mm}$-radius spheres around the peak voxels reported in a previous, separate study (Brown et al., 2006). Brown and colleagues'(2006) study was selected because 1) it was the study where we adapted the block-design version of the Go/No-Go task from and 2) we used similar acquisition parameters (e.g., Siemens 3T scanner, TR, TE and FOV) and image processing (e.g., SPM) to their study. We extracted the percent signal change within the IFG and ACC during the

contrast of Go/No-Go relative to Go conditions using Marsbar software (http://marsbar.sourceforge.net/).

\subsubsection{Psychophysiological interaction analyses:}

The psychophysiological interaction (PPI) analysis (Friston et al., 1997; O'Reilly, Woolrich, Behrens, Smith, \& Johansen-Berg, 2012) were implemented to examine the regions that 


\section{Running Head: CULTURE AND NEURAL BASIS OF INHIBITORY CONTROL}

had stronger task-dependent functional connectivity with the IFG, such that the connectivity was stronger during the Go/NoGo blocks compared to the Go blocks. Similar to our ROI analysis, we created a seed region using a $6 \mathrm{~mm}$ sphere around the IFG area as defined in Brown and colleagues'(2006) study. At the first-level analysis, we applied the generalized PPI toolbox implemented in SPM8 as follows (Gitelman, Penny, Ashburner, \& Friston, 2003; McLaren, Ries, Xu, \& Johnson, 2012). First, we extracted the first eigenvariate time series within the IFG seed. We then created two separate PPI terms using element-by-element products of the extracted, deconvolved IFG time series and each task regressor [Go/No-Go and Go]. These two PPI terms were then reconvolved with the canonical hemodynamic response function and entered as regressors (PPI regressor) along with the task regressors (psychological regressors), IFG eigenvariate time series (physiological regressor), and linear regressor that accounted for lowfrequency drift (regressor of no-interest). The contrast between the PPI regressors [Go/No-Go > Go] for each participant was then used at the second-level analysis. Similar to the whole-brain analysis without PPI regressors, here we employed the 3dClustSim method to keep a clusterwise threshold of $p<0.05$.

Because PPI analyses are known to lack statistical power, and tend to exhibit a high level of false negatives (O'Reilly et al., 2012), we focused our second-level analysis on the consistent pattern of PPIs across the whole participants (but see Supplementary Text, Supplementary Table 1 and Supplementary Figure 3 for the differences in PPIs as a function of cultural groups). Using the whole samples should allow sufficient power to detect and reveal connectivity between the IFG and other areas during the Go/No-Go task, thereby providing insights into the role of the IFG.

\section{Results}

\subsection{Behavioral results}

\subsubsection{Behavioral performance and cultural groups}




\section{Running Head: CULTURE AND NEURAL BASIS OF INHIBITORY CONTROL}

A [2 (Go/No-Go vs. Go blocks) X 3 (Caucasian Americans vs. Japanese Americans vs. Native Japanese)] mixed-design ANOVA on reaction times (Table 1) revealed a significant effect of blocks such that reaction times were slower during the Go/No-Go blocks $(M=400.38 \mathrm{~ms}, S D=$ $54.38)$ than during the Go blocks $\left(M=365 \mathrm{~ms}, S D=47.6 ; F(1,55)=57.44, p<.001, \eta_{p}{ }^{2}=.51\right)$. There were no significant effects of cultural groups $\left(F(2,55)=1.56, p=.22, \eta_{p}{ }^{2}=.05\right)$ or interaction $\left(F(2,55)=.125, p=.88, \eta_{p}^{2}=.005\right)$ on reaction times.

Similarly, a $[2$ (Go/No-Go vs. Go blocks) X 3 (Caucasian Americans vs. Japanese Americans vs. Native Japanese)] mixed-design ANOVA on accuracy rates (Table 1) revealed a significant effect of blocks such that accuracy rates were worse during the Go/No-Go blocks $(M=$ $96.01 \%, S D=3.73)$ than during the Go blocks $(M=99.51 \%, S D=1.71 ; F(1,55)=57.08, p<.001$, $\left.\eta_{p}{ }^{2}=.51\right)$. There were no significant effects of cultural groups $\left(F(2,55)=0.87, p=.92, \eta_{p}{ }^{2}=.003\right)$ or interaction $\left(F(2,55)=2.26, p=.11, \eta_{p}^{2}=.08\right)$ on accuracy rates. Consistent with previous studies (Brown et al., 2006; Horn et al., 2003), generally participants' accuracy rates in the entire task [e.g., hit rate $(M=98.71 \%, S D=1.13)$, false alarm $(M=7.68 \%, S D=6.47)$ ] were near ceiling in this version of a Go/No-Go task.

Given our focus on the [Go/No-Go $>$ Go] contrast in our fMRI analysis, we also calculated the difference in reaction times and accuracy rates by subtracting these two indices in the Go blocks from those in the Go/No-Go blocks. The difference in reaction times was marginally, negatively correlated with the difference in accuracy rates $(r(56)=-.24, p=.07)$. This suggests a possibility of speed-accuracy trade-off in the task.

\subsubsection{Cultural groups and behavioral consistency}

There was a significant effect of cultural groups on behavioral consistency $(F(2,55)=$ $\left.9.36, p<.001, \eta_{p}{ }^{2}=.25\right)$. Consistent with previous cultural research that used different operational definition of behavioral consistency (Kanagawa et al., 2001), Caucasian Americans showed significantly higher behavioral consistency compared to Native Japanese $(p<.001$, Tukey HSD corrected, Table 1). Japanese Americans' behavioral consistency was in between that of the other 
Running Head: CULTURE AND NEURAL BASIS OF INHIBITORY CONTROL

groups but was not significantly different from either group $(p$ 's $>.09){ }^{3}$

\subsubsection{Behavioral performance, cultural groups and behavioral consistency}

Behavioral consistency was not correlated with reaction times in either the Go block or Go/No-Go block as well as with the difference in reaction times between the two blocks ( $p$ 's $>.11)$. Similarly, there was no significant relationship between behavioral consistency and accuracy rates in either the Go or Go/No-Go block as well as the difference in accuracy rates between the two blocks $(p$ 's $>.68)$. There were also no interactions between behavioral consistency and cultural groups on any behavioral performance indices $(p$ 's $>.30)$.

\footnotetext{
${ }^{3}$ For completeness, we also considered the Self-Construal Scale (SCS) as having a two-factor
} structure: Independent and Interdependent factors (Singelis, 1994). While there was no significant effect of cultural groups on Interdependent scores $\left(F(2,55)=2.41, p=.10, \eta_{p}{ }^{2}=.08\right)$, there was an effect of cultural groups on Independent scores $\left(F(2,55)=3.90, p=.03, \eta_{p}{ }^{2}=.12\right)$.

Particularly, Caucasian Americans $(M=4.93, \mathrm{SD}=.85)$ showed significantly higher Independent scores than Japanese Americans $(M=4.2, \mathrm{SD}=.66, p=.03$, Tukey HSD corrected). Caucasian Americans's Independent scores were marginally higher than Native Japanese ( $M=4.51$, SD $=.60)$ 's $(p=.06$ without Tukey HSD correction and $p=.14$ with Tukey HSD correction). There was no significant difference in Independent scores between Japanese Americans and Native Japanese ( $p=.35$ without Tukey HSD correction, $p=.62$ with Tukey HSD correction).

It is important to note that while this pattern of SCS scores did not totally match the expected pattern among cultural groups based on original conceptualization of the SCS (e.g., Interdependent scores should be lower among Caucasian Americans) (Singelis, 1994), this inconsistency is relatively common in cross-cultural research (Oyserman, Coon, \& Kemmelmeier, 2002). A number of studies, reviews and meta-analyses have shown this inconsistency pattern between cultural-group (e.g., East Asians and Caucasian Americans) and cultural-value (SCS) levels of analysis (Kitayama, Park, Sevincer, Karasawa, \& Uskul, 2009; Levine et al., 2003; Matsumoto, 1999). For instance, in a meta-analysis, Caucasian Americans were not found to be higher in their Interdependent scores than East Asians (Levine et al., 2003). Many possible explanations were given to remedy these inconsistencies (for review see, Cross et al., 2011). For instance, it is possible that cultural-group and cultural-value levels of analyses operate differently (i.e., one at the societal level and the other at the individual level) and may not always be related to each other (Kitayama et al., 2009; Oyserman \& Uskul, 2008; van de Vijver, van Hemert, \& Poortinga, 2008). Another possible explanation is that two board dimensions of SCS (i.e., Interdependent and Independent dimensions) may not capture specific aspects of selfconstrue that are most likely to vary cross-culturally (Noguchi, 2007; Somech, 2000). It has been suggested, therefore, to focus on specific factors (such as, behavioral consistency) of the SCS rather than on the broader dimensions (Cross et al., 2011; Hardin, 2006; Hardin et al., 2004). The current research follows these two views by 1) separately investigating cultural-group and cultural-value levels of analyses and 2) focusing on a specific factor of the SCS (i.e., behavioral consistency). 


\section{Running Head: CULTURE AND NEURAL BASIS OF INHIBITORY CONTROL}

\subsection{Neuroimaging results}

\subsubsection{Whole-brain analyses:}

Consistent with previous studies (e.g., Buchsbaum et al., 2005; Criaud \& Boulinguez, 2013; Simmonds et al., 2008; Swick et al., 2011), across participants, greater neural activity within the bilateral IFG and ACC as well as other areas, such as precentral gyrus, right superior frontal gyrus (R-SFG), and superior temporal gyrus (STG), was observed in the Go/No-Go condition compared to the Go condition (Table 2, Supplementary Figure 1).

Next, we examined variation in inhibitory-control brain activity at the cultural-group level (Table 3). Consistent with our hypothesis, the culture-driven set of contrasts revealed that Native Japanese participants elicited greater reactivity that surpassed our 3dClustSim-corrected cluster extent threshold in the left-IFG (L-IFG, Figure 2a) than Caucasian American and Japanese American participants did. Caucasian American and Japanese American participants, on the contrary, elicited greater reactivity in other areas, such as bilateral precentral gyrus and right culmen in the cerebellum, than Native Japanese counterparts did. As for the race-driven set of contrasts, no supra-threshold clusters were found. That is, there was no statistical difference in inhibitory-control neural responses between Caucasian-American participants and those of Japanese descent (Japanese Americans and Native Japanese).

As for variation in inhibitory-control brain activity at the cultural-value level, a wholebrain regression analysis with the $[\mathrm{Go} / \mathrm{No}-\mathrm{Go}>\mathrm{Go}]$ contrast across cultural groups with behavioral consistency as a covariate of interest was conducted using a 3dClustSim-corrected cluster extent threshold. In line with prediction, we found a significant, positive relationship between behavioral consistency and the degree of neural activity in the left hemisphere within a region cluster in the rostral ACC (rACC) that extended to the medial frontal gyrus (Table 3, Figure 3a). 


\section{Running Head: CULTURE AND NEURAL BASIS OF INHIBITORY CONTROL}

\subsubsection{A-priori region of interest analyses:}

Given that the effects of culture on the L-IFG and ACC were already emerged from the clusterwise-corrected whole-brain analyses, our ROI analyses were conducted to follow-up the whole-brain analyses. One-way ANOVA within the independently defined L-IFG area $[-32,25,-1]$ (Brown et al., 2006) revealed a significant cultural-group effect $\left(F(2,55)=3.71, p=.03, \eta_{p}{ }^{2}=.12\right.$, Figure $2 b$ ). Nonetheless, there is a possibility that variances of the percent signal change within the L-IFG was unequal across cultural groups, given that Levene's test (Levene, 1960) was marginally significant $(\mathrm{F}(2,55)=2.78, p=.07)$. Hence, in addition to using one-way ANOVA, we also employed Mood's Median test that has no assumption about data distribution and does not assume homogenetiy of variances (Siegel, 1956). Similar to the result from one-way ANOVA, Mood's Median test revealed a signficant cultural-group effect $\left(\chi^{2}(2, \mathrm{~N}=58)=6.91, p=.03\right)$. Specifically, L-IFG activity of Native-Japanese participants $(M d n=.08, I Q R=.17)$ was significantly higher than that of Japanese American participants $(M d n=-.04, I Q R=.17, p=.009)$ and that of Caucasian American participants $(M d n=.03, I Q R=.13, p=.04)$. There was no difference in L-IFG activity between Caucasian American and Japanese American participants $(p=.22)$. Hence, this pattern of L-IFG activity among cultural groups is in line with the culture-driven hypothesis (as opposed to the race-driven hypothesis). Behavioral consistency did not correlate with L-IFG activity $(r=-.11$, $p=.41)$. Moreover, there was no interaction between cultural groups and behavioral consistency on L-IFG activity $\left(\mathrm{F}(2,52)=.02, p=.98, \eta_{p}{ }^{2}=.001\right)$ when cultural groups, behavioral consistency and their interaction were entered in the same model. Additionally, L-IFG activity did not correlate with any behavioral performance indices, including the differences in RT and accuracy-rate between blocks [Go/No-Go blocks - Go blocks] ( $p$ 's $>$.66).

For the ACC, when the ROI was defined using the peak voxels reported in Brown and colleagues‘ (2006) study [-2, 23, 39], we found a significant, albeit small, positive correlation between ACC activity and behavioral consistency $(r(56)=.26, p=.05)$. Similar correlational 
Running Head: CULTURE AND NEURAL BASIS OF INHIBITORY CONTROL

magnitudes were also found when the ACC was defined based on a recent larger-scale event-related fMRI Go/NoGo study (Steele et al., 2013): $[6,27,33](r(56)=.28, p=.03)$ and $[3,26,32](r(56)$ $=.29, p=.03$ ). These patterns of correlations suggest that the more consistent participants' behaviors were across situations, the greater ACC activity the participants elicited. On closer examination, however, the ACC coordinates from these two studies (Brown et al., 2006; Steele et al., 2013) were at the dorsal part of the ACC (dACC; Supplementary Figure 2), while the effect at the cultural-value level emerged from the whole-brain analyses was mainly at the rostral part of the ACC (rACC; Figure 3a). Accordingly, we employed another independently defined ROI for the rACC using the peak voxels produced by a whole-brain, one-sample t-test analysis with [Go/NoGo $>$ Go] contrast collapsing across all participants $[-3,33,15]$ (Supplementary Figure 2).

Consistent with the results from the whole-brain analyses and ROI analyses using dACC coordinates (Brown et al., 2006; Steele et al., 2013), an ROI analysis using an independentlydefined rACC coordinate ${ }^{4}$ reveals that rACC activity correlated positively with behavioral consistency $\left(r(56)=.44, p=.001 ; r_{s}(56)=.48, p=.001 \text {, Figure } 3 b\right)^{5}$, with a numerically higher magnitude than when dACC was used. ${ }^{6}$ Moreover, rACC activity was also correlated with

\footnotetext{
${ }^{4}$ Given that this rACC ROI was identified by the peak voxels of a whole-brain, one-sample t-test analysis with [Go/No-Go $>$ Go] contrast collapsing across all participants, it should be independent from the any effects based on individual differences among participants. That is, the whole-brain, one-sample t-test analysis only showed that this region was significantly stronger during the Go/NoGo blocks than during the Go blocks across every participant. However, it did not indicate that the Go/No-Go > Go effect would vary as a function of variables related to individual-differences among participants, such as behavioral consistency.

${ }^{5}$ Upon inspection of the data, nonetheless, there appeared to be some potential outliers in the rACC percent signal change. Hence, in addition to reporting Pearson's correlation $(r)$, we also reported Spearman's Rho $\left(r_{s}\right)$ that is less influenced by outliers, but has less statistical power. ${ }^{6}$ For completeness, we also considered the Self-Construal Scale (SCS) as having a two-factor structure (Singelis, 1994). Both the IFG and rACC activity did not correlate with either interdependent $(p ' s>.25)$ or independent $(p ' s>.23)$ scores. This finding may reflect that the two-factor structure of the SCS is not adequate for capturing the specific dimension of cultural values (Cross et al., 2011; Levine et al., 2003). Additionally, when a multiple-factor structure of the SCS was considered, additional five factors in the SCS (beside behavioral consistency) were also computed (Hardin, 2006; Hardin et al., 2004). Similarly, both the IFG and rACC activity did
} 


\section{Running Head: CULTURE AND NEURAL BASIS OF INHIBITORY CONTROL}

behavioral performance indices, including, firstly, the reaction-time (RT) difference between blocks [RT in Go/No-Go blocks - Go blocks] $\left(r(56)=.27, p=.04 ; r_{s}(56)=.26, p=.05\right.$, see Figure 3c), such that the greater rACC activity, the slower the participants. Secondly, rACC activity was marginally correlated with the accuracy-rate difference between blocks [accuracy rate in Go/NoGo blocks - Go blocks] $\left(r(56)=-.35, p=.007 ; r_{s}(56)=-.24, p=.07\right.$, Figure 3d), such that greater rACC activity was associated with worse accuracy in the task. Nonetheless, there was no effect of cultural groups on rACC activity $\left(F(2,55)=.43, p=.65, \eta_{p}{ }^{2}=.02\right)$. The interaction between cultural groups and behavioral consistency on rACC activity was also not significant $(F(2,52)=$ $\left..86, p=.43, \eta_{p}{ }^{2}=.03\right)$ when cultural groups, behavioral consistency and their interaction were entered into the same model.

\subsubsection{Psychophysiological interaction analyses:}

While both whole-brain and ROI analyses showed that L-IFG activity was stronger among Native Japanese participants compared to other cultural groups, unlike rACC activity, L-IFG activity did not correspond to behavioral indices. Accordingly, we employed the PPI analysis (Friston et al., 1997; O'Reilly et al., 2012) to further investigate the role of the L-IFG in the current study. Specifically, implementing a simple t-test across subjects, we found task-dependent functional connectivity between L-IFG activity and activity in the prefrontal cortex that extended to the rACC, medial frontal gyri and superior frontal gyri (see Table 4, Figure 4). That is, L-IFG activity covaried with prefrontal activity during the Go/No-Go blocks more than during the Go blocks.

not significantly correlate with these additional five factors $(p$ 's $>.2)$. This suggests that the cultural-value effect at rACC was specific to behavioral consistency. Note that the shorter version of the SCS (24 items) was used here, compared to the longer version (30 items) (Hardin, 2006; Hardin et al., 2004). Although the short version has all behavioral consistency items, some of the items for additional five factors were missing. Future research that wishes to investigate other factors may need to employ the longer version of the scale. 


\section{Running Head: CULTURE AND NEURAL BASIS OF INHIBITORY CONTROL}

\section{Discussion}

Over the past decades, cognitive neuroscientists have conducted extensive research on neural basis of inhibitory control, using different populations at various parts of the world (e.g., Buchsbaum et al., 2005; Criaud \& Boulinguez, 2013; Simmonds et al., 2008; Swick et al., 2011). At the same time, cultural psychologists have started to support the notion that culture has an influence on inhibitory control (e.g., Coffey et al., 2005; Matsumoto et al., 2005; Oh \& Lewis, 2008; Razani et al., 2007). However, to the best of our knowledge, no neuroimaging research has yet systematically investigated the influence of culture on inhibitory-control brain activity. Such neural investigation may lead to better understanding of specific neural processes that underlie the cultural differentiation in inhibitory control (Cheon, Wang, Chiao, Tang, in press). Here we show, for the first time, the extent to which culture at both cultural-group and cultural-value levels of analysis modulated inhibitory control brain activity. Importantly, the effect of cultural groups seemed to be most prominently at the L-IFG, while the effect of a cultural value (i.e., behavioral consistency) appeared concentrated at the rACC. Based on our ROI analyses, the effect of cultural groups at the rACC and a cultural value at the L-IFG were not significant and had very small effect sizes. Thus, it is unlikely that the differential effects found (i.e., the effect of cultural groups at the IFG, not rACC, and the effect of a cultural value at the rACC, not IFG) were due mainly to low statistical power. We argue instead that these may reflect how cultural groups and a cultural value may modulate different aspects of inhibitory-control brain activity. This is consistent with previous cultural neuroscience studies showing neural variation as a function of cultural values, irrespective of cultural groups, and vice versa (e.g., Chiao et al., 2009b; Chiao et al., 2010; de Greck et al., 2012; Han et al., 2013; Sul et al., 2011). Nonetheless, it is important to note that our PPI results showed the connectivity between the L-IFG and rACC during the Go/No-Go blocks more than during the Go blocks. This suggests that, although cultural groups and values may differentially influence different areas of the inhibitory-control network, these two areas may still work together in an inhibitory-control situation. 


\section{Running Head: CULTURE AND NEURAL BASIS OF INHIBITORY CONTROL}

\subsection{Cultural-group level of analysis}

At the cultural-group level of analysis, we tested whether cultural variation in inhibitorycontrol brain activity is due to culture or race. Our results with IFG activity support the culturedriven, but not the race-driven, hypothesis. This is given that IFG activity was explained by culture where one lived in as opposed to race. Specifically, native Japanese participants elicited stronger L-IFG activity in the Go/NoGo task than those living in the US regardless of whether they were Caucasian or Japanese Americans. L-IFG activity among Caucasian participants, on the other hand, was not systematically different from those of Japanese descent. Previous neuroimaging and lesion studies have suggested the essential role of the L-IFG in inhibitory control (Chuah et al., 2006; Swick et al., 2011). Not only did patients with L-IFG lesions who had R-IFG intact perform poorly on the Go/No-Go task (Swick et al., 2008), but activity in the L-IFG was also correlated with the efficiency of the task performance in terms of both RT and accuracy in a more difficult version of a Go/No-Go task (Hirose et al., 2012), in addition to being correlated with developmental maturity (Tamm et al., 2002).

In the current study, whereas the L-IFG did not respond to behavioral indices, our PPI results across all participants revealed a task-dependent functional connectivity between the L-IFG and several areas in the prefrontal cortex, including the rACC and medial and superior frontal gyri. This suggests that when there was a high demand for inhibitory control (i.e., during the Go/No-Go block), activity in the L-IFG covaried with activity in other prefrontal areas in the inhibitory network, confirming the pivotal role of the L-IFG in inhibitory control. Still, it is difficult to interpret the meaning of higher activation of the L-IFG among native Japanese participants. While we could not conclude for certain that higher activation of the L-IFG means higher efficiency in inhibitory control, it should still be appropriate to infer that cultural group affiliation (as opposed to race) modulated L-IFG activity during inhibitory-control processes. Our L-IFG finding is consistent with Lahat and colleagues' (2010) developmental ERP Go/No-Go study in 5 year-old children where the effect of culture on inhibitory control was found neurally (ERP N2 component) 


\section{Running Head: CULTURE AND NEURAL BASIS OF INHIBITORY CONTROL}

but not behaviorally. In fact, the location of their N2 component through an ERP sourcelocalization algorithm is closely matched with the location of our IFG, providing convergent evidence that the neural effect of cultural-group affiliation may emerge in the absence of behavioral differences.

\subsection{Cultural-value level of analysis}

At the cultural-value level of analysis, we focused on the cultural value of behavioral consistency, and tested whether behavioral consistency modulated inhibitory control activity. Firstly, Caucasian Americans' behavioral consistency was highest, while Native Japanese's was lowest. This pattern of behavioral consistency across cultural groups as operationalized by a selfreport questionnaire in the current study (Hardin, 2006; 2004) is in line with the pattern in previous research that operationalized behavioral consistency as self-description across situations (Kanagawa et al., 2001). This consistency makes it likely that the similar construct of behavioral consistency was accessed across studies. More importantly, across individuals and cultural groups, behavioral consistency was positively correlated with rACC activity in the Go/NoGo task. Activity in the ACC is reliably elicited by the Go/No-Go task (Steele et al., 2013), and is stronger when people experience high conflict in the task (Braver, Barch, Gray, Molfese, \& Snyder, 2001; Fassbender et al., 2004; Kiehl, Liddle, \& Hopfinger, 2000; Menon, Adleman, White, Glover, \& Reiss, 2001). Moreover, in the present study, activity in the rACC region was also associated with reduced performance in the task in terms of both slower RT and poorer accuracy. This suggests that strong neural responses of conflicts within the rACC region, in turn, resulted in weakening efficiency of inhibitory control. Altogether our rACC results suggest that those who value being "themselves" across situations (i.e., having higher endorsement of behavioral consistency) may be less efficient in their inhibitory-control processes. This is perhaps because high behavioral consistency individuals lack the opportunity for switching cognitive mindsets across situations in their daily lives. Our results are consistent with cultural acculturation findings in which those who 


\section{Running Head: CULTURE AND NEURAL BASIS OF INHIBITORY CONTROL}

are less flexible to adapt to the changes in new cultural contexts are less proficient in inhibitory control tasks (Coffey et al., 2005; Razani et al., 2007).

\subsection{Future directions}

It is our hope that the current study may set a foundation for future cultural neuroscience research to further investigate the complex modulatory roles of culture on the neural basis of inhibitory control. One aspect that is left unclear in our study is the cognitive aspect of the IFG and ACC. While we demonstrated that the IFG and ACC during inhibitory control were modulated by cultural-group affiliation and cultural value of behavioral consistency, respectively, we could not pinpoint the exact cognitive roles of these areas. Our PPI results revealed that these two areas were more functionally connected with each other during the Go/No-Go blocks, and previous research reliably found an enhanced activity in these two areas in various inhibitory-control tasks (Buchsbaum et al., 2005; Criaud \& Boulinguez, 2013; Simmonds et al., 2008; Swick et al., 2011). More specifically, the IFG is generally linked to response inhibition (Swick et al., 2011), while the ACC is related to conflict-monitoring (Braver et al., 2001). It is possible, for instance, that higher L-IFG activity among Native Japanese may reflect stronger response inhibition due to their social norm emphasizing self-control (Chao \& Tseng, 2002; Kwon, 2002; Wang \& Mao, 1996). On the other hand, the relationship between high behavioral consistency and stronger rACC activity may reflect stronger conflict-monitoring among those who had limited opportunities to practice their inhibitory control (Coffey et al., 2005; Razani et al., 2007). Accordingly, future cultural research may further manipulate these two cognitive aspects of inhibitory control (response inhibition and conflict monitoring), and investigate the effects such manipulation has on the IFG and ACC. For instance, future studies can compare between pressing to Go stimuli (high response inhibition) and counting Go stimuli (low response inhibition) (Smith, Johnstone, \& Barry, 2008). As for conflictmonitoring, future research can alter the ratio of target to non-target stimuli where the fewer nontarget stimuli, the more conflict it is (Nieuwenhuis, Yeung, Van Den Wildenberg, \& Ridderinkhof, 2003). 


\section{Running Head: CULTURE AND NEURAL BASIS OF INHIBITORY CONTROL}

Another direction is to incorporate social aspects to an inhibitory-control situation. Cultural psychology research has argued that cultural differences in inhibitory control are partly due to differences in social norms. Specifically, in order to conform to social norms of collectivistic culture, East Asians are more likely to suppress their action when interacting with external environment, compared to Westerners (Ekman, 1971). One example is their inhibition of emotional expression in which Japanese adults, compared to American adults, were more likely to report expressing their feelings with less intensity than their true feelings (Matsumoto et al., 2005). Moreover, such inhibition of expression is especially pronounced when the experimenters were in a room with Japanese participants, compared to when the Japanese participants were alone by themselves (Ekman, 1971). Future cultural studies may, for instance, add social feedback to a Go/No-Go task, and see how such feedback influences inhibitory-control brain activity. Additionally, future research may further our understanding of the role of culture on inhibitorycontrol brain activity by investigating possible mediating factors. Among potential candidates that may mediate the relationship are motivation-related traits. Cultural psychology research has related the cultural dimension of individualism and collectivism with individual-differences in performance goal and approach/avoidance motivation (Dekker \& Fischer, 2008; Tanaka \& Yamauchi, 2004; Urdan, 2004). For instance, Tanaka and Yamaguchi (2004) found that among Japanese participants, higher independent scores from the Self-Construal Scale (SCS) (Singelis, 1994) positively predicted their approach motivation (BAS) while negatively predicted their avoidance motivation (BIS) based on the Behavioral Inhibition/Activation System (BIS/BAS) scale (Carver \& White, 1994). Hence, it is possible that living in a collectivistic/individualistic culture, and/or having high/low behavioral consistency may influence one's motivation while performing an inhibitory-control task that results in differences in brain activity. Unfortunately, measures related to motivation were not collected in our study, and thus this possibility needs to be tested more formally in future research.

\section{4 Limitations}




\section{Running Head: CULTURE AND NEURAL BASIS OF INHIBITORY CONTROL}

This study is not without limitations, however. First as mentioned earlier and elsewhere (Brown et al., 2006; Horn et al., 2003), this block-design version of a Go/NoGo task is not difficult. It was designed to have high statistical power than the event-related design version, so that it can detect subtle cultural phenomena at the neural level (Brown et al., 2006). The possible cost to this current study is that we found neural variation in the hypothesized areas that may not necessarily predict variation in inhibitory behavior. This is perhaps due to the ceiling effect in behavioral performance, especially in terms of accuracy. Cultural differentiation in neural responses in the absence of behavioral responses has previously been observed not only during inhibitory control, but also during other situations, such as social cognition (e.g., Chiao et al., 2009a; Lahat et al., 2010). Whether this discrepancy reflects a design confound or, instead, the closer proximity from the brain to cultural phenomenon (Chiao, Cheon, Pornpattananangkul, Mrazek, \& Blizinsky, 2013) awaits future research examining cultural influences on neural basis of inhibitory control, with more challenging inhibitory-control tasks that elicit broader behavioral variation. Another limitation of the Go/No-Go task in this study is the nature of a block design, as it may not allow for isolation of inhibitory processes from other overlapping executive control processes, e.g. attention and association between stimulus and response (Criaud \& Boulinguez, 2013; Simmonds et al., 2008). For instance, it remains unknown whether behavioral variation is associated with neural variation during the inhibition task for each study trial. Nonetheless, the block design may recruit a "core system" underlying the maintenance of a controlling task set over time (Dosenbach et al., 2006; Swick et al., 2011). Such a process is important for inhibitory control and may resemble changes in cultural situations, but may not be found with an event-related design (Dosenbach et al., 2006). Moreover, the PPI analyses conducted here are more suitable to a block design than an event-related design because a block design relies less on the assumption of the hemodynamic response function shape (O’Reilly et al., 2012).

Another limitation is the difference in language used in stimuli across cultural groups. English letters were employed for Caucasian American and Japanese American participants, while 


\section{Running Head: CULTURE AND NEURAL BASIS OF INHIBITORY CONTROL}

Japanese letters were utilized for Native Japanese participants. This is because the English-letter version of the task has been well validated and used in the past to investigate individual differences in inhibitory-control brain activity among English-speaking participants (Brown et al., 2006; Horn et al., 2003). To relate our results to this well-established literature, we decided to use the same task and developed an equivalent version of the task that is specific to Japanese speakers. However, it is possible that the cultural variation in the L-IFG found in the current study could be accounted for by language. Yet, we argue that this is unlikely, given that (1) both English letters and Japanese Katakana letters are both phonogram, (2) they are native language letters for each group of participants, (3) the two non-target letters “v" and "レ" were similar in both geometry and the frequency of their usage (Chikamatsu et al., 2000; Lewand, 2000; http://www.oxforddictionaries.com). Moreover, (4) our study implemented the subtraction paradigm by comparing contrasts based upon differences in brain activity between Go/No-Go and Go conditions. Given that the same language was used for both Go/No-Go and Go conditions, the contrast of Go/No-Go relative to Go conditions should already control the influence of language. To further test the influence of languages used, we conducted whole-brain analyses with the Go condition only, using the same sets of one-way ANOVA contrasts with the main Go/NoGo > Go analysis at the cultural-group level (see Supplemental Table 2). We did not see any significant effect at the L-IFG, suggesting that our main cultural-group results at the L-IFG did not driven by differences in a language used. Yet, future research should control for the influence of language using the same target/non-target symbols across groups of participants.

\subsection{Conclusion}

In conclusion, we identified cultural influences on neurobiological mechanisms of inhibitory control, a primary building block to complex cognition. At the cultural-group level, we demonstrate for the first time that living in a collectivistic culture where high self-control is valued is associated with higher L-IFG activity during behavioral inhibition relative to those living in an 


\section{Running Head: CULTURE AND NEURAL BASIS OF INHIBITORY CONTROL}

individualistic culture. Importantly, such cultural variation in L-IFG activity cannot be explained by differences across racial groups. At the cultural-value level, we discovered that cultural values of behavioral consistency or emphasizing inflexibility in one's behaviors across contexts is associated with higher rACC activity during inhibition. Altogether, our findings provide novel insight into the neurobiological basis of cultural differences in behavioral inhibition (Ekman, 1971; Matsumoto et al., 2005). Our study may provide a foundation for future research examining the cultural and neurobiological processes underlying inhibitory control across social and ecological contexts. 


\section{Running Head: CULTURE AND NEURAL BASIS OF INHIBITORY CONTROL}

Acknowledgements. We thank Paul Reber, Bobby Cheon, Lisa Hechtman, Christina Young, Nicolas Escoffier and Vivian Wang for helpful comments, Donna Bridge and Trixie Lipke for their assistance in data collection, and Jason Scimeca and Mark Schurgin for technical assistance. This work was funded in part by National Science Foundation grants BCS-0720312 and BCS-0722326 and the Japan Society for the Promotion of Science Fellowship to J.Y.C, a Fulbright Open Competition Scholarship Program, and NIH grant T32 NS047987 to N.P, the Grants-in Aid for

Scientific Research S\#21220005 from the Japan Society for the Promotion of Science and the "Development of biomarker candidates for social behavior" grant from the Ministry of Education, Culture, Sports, Science and Technology grant to N.S. and the KAKENHI No. 20020011 and Nakayama Foundation for Human Science grants to T.I. 
Running Head: CULTURE AND NEURAL BASIS OF INHIBITORY CONTROL

Table 1. Means of behavioral results. Standard deviations are in the parentheses.

\begin{tabular}{|c|c|c|c|}
\hline & Caucasian Americans & Japanese Americans & $\begin{array}{l}\text { Native } \\
\text { Japanese }\end{array}$ \\
\hline Go/No-Go block RT (ms) & $415.13(65.59)$ & $392.25(45.10)$ & $392.88(48.41)$ \\
\hline Go block RT (ms) & $380.54(56.84)$ & $356.66(51.52)$ & $354.74(31.11)$ \\
\hline Go/No-Go block Accuracy Rate (\%) & $95.58(3.72)$ & $96.98(3.73)$ & $95.68(3.80)$ \\
\hline Go block Accuracy Rate (\%) & $99.75(.61)$ & $98.96(3.01)$ & $99.70(.66)$ \\
\hline Go/No-Go block d' & $3.75(.58)$ & $4.04(.53)$ & $3.81(.60)$ \\
\hline Behavioral consistency & $4.73(1.08)$ & $3.91(1.21)$ & $3.20(1.13)$ \\
\hline
\end{tabular}


Running Head: CULTURE AND NEURAL BASIS OF INHIBITORY CONTROL

Table 2. Neural correlates of inhibitory control $[\mathrm{Go} / \mathrm{No}-\mathrm{Go}>\mathrm{Go}]$ on the whole sample. A $3 \mathrm{dClustSim}$-corrected cluster extent threshold $(p<0.005$, clusterwise corrected at $p=.05)$ was used. Coordinates were listed in MNI. BA denotes Brodmann Area.

\begin{tabular}{l|l|l|l|l|l|l}
\hline$X$ & $Y$ & \multicolumn{1}{l}{ B } & \multicolumn{1}{l}{ BA } & \multicolumn{1}{l}{ Voxels } & Brain area \\
\hline 60 & -48 & 15 & 8.77 & 22 & & R Superior Temporal Gyrus \\
39 & 21 & -9 & 7.41 & 47 & 3000 & R Inferior Frontal Gyrus \\
-30 & 18 & -15 & 5.55 & 47 & & L Inferior Frontal Gyrus \\
\hline-60 & -54 & 15 & 6.07 & 22 & 375 & L Superior Temporal Gyrus \\
\hline-9 & 27 & 27 & 5.7 & 32 & 904 & Dorsal Anterior Cingulate Cortex \\
-3 & 33 & 15 & 4.89 & 24 & & Rostral Anterior Cingulate Cortex \\
\hline-3 & -18 & 36 & 5.56 & 24 & 139 & Posterior Cingulate Cortex \\
\hline 48 & -6 & 57 & 4.52 & 6 & 69 & R Precentral Gyrus \\
\hline-9 & -84 & 3 & 4.46 & 17 & 190 & L Cuneus \\
\hline 36 & 51 & 27 & 4.10 & 10 & 104 & R Superior Frontal Gyrus \\
\hline
\end{tabular}




\section{Running Head: CULTURE AND NEURAL BASIS OF INHIBITORY CONTROL}

Table 3. Variation in inhibitory-control brain activity [Go/No-Go $>$ Go] at the cultural-group level (a one-way ANOVA between cultural groups, using two sets of planed contrasts to test the culturedriven and race-driven hypotheses) and at the cultural-value level (a regression analysis with behavioral consistency as the covariate of interest). A 3dClustSim-corrected cluster extent threshold $(p<0.005$, clusterwise corrected at $p=.05)$ was used. Coordinates were listed in MNI. Regions shown in figures were bold. BA denotes Brodmann Area.

\begin{tabular}{lllllll}
\hline$X$ & $Y$ & $Z$ & $t$ & BA & Voxels & Brain area \\
\hline
\end{tabular}

\section{The cultural-group level:}

1) Culture-driven ANOVA contrasts:

Native Japanese > (Caucasian Americans \& Japanese Americans)

\begin{tabular}{l|l|l|l|l|l|l}
-39 & 24 & 3 & 4.56 & 47 & 46 & L Inferior Frontal Gyrus
\end{tabular}

(Caucasian Americans \& Japanese Americans) > Native Japanese

\begin{tabular}{l|l|l|l|l|l|l}
18 & -51 & -15 & 4.82 & & 108 & R Culmen in Cerebellum \\
\hline-30 & -18 & 57 & 4.08 & 4 & 120 & L Precentral Gyrus \\
\hline 39 & -15 & 48 & 4.01 & 6 & 133 & R Precentral Gyrus \\
\hline 9 & -24 & 51 & 3.72 & 6 & 41 & Paracentral Lobule
\end{tabular}

2) Race-driven ANOVA contrasts:

(Native Japanese \& Japanese Americans) > Caucasian Americans

no supra-threshold clusters were found

Caucasian Americans > (Native Japanese \& Japanese Americans)

no supra-threshold clusters were found

$\underline{\text { The cultural-value level: }}$

1) Positive Correlation with Behavioral Consistency

\begin{tabular}{l|l|l|l|l|l|l}
-6 & 36 & 12 & 5.66 & 32 & 465 & Rostral Anterior Cingulate Cortex \\
\hline 24 & 51 & 33 & 3.69 & 9 & 53 & R Superior Frontal Gyrus \\
\hline 57 & -51 & 30 & 3.55 & 40 & 51 & R Supramarginal Gyrus
\end{tabular}

2) Negative Correlation with Behavioral Consistency no supra-threshold clusters were found 
Running Head: CULTURE AND NEURAL BASIS OF INHIBITORY CONTROL

Table 4. Neural regions showing stronger connectivity with the L-IFG $[-32,25,-1]$ (Brown et al., 2006) during the Go/No-Go blocks, compared to Go blocks on the whole sample. A 3dClustSimcorrected cluster extent threshold $(p<0.005$, clusterwise corrected at $p=.05)$ was used. Coordinates were listed in MNI. BA denotes Brodmann Area.

\begin{tabular}{l|l|l|l|l|l|l}
\hline$X$ & \multicolumn{1}{c}{$Y$} & \multicolumn{1}{c}{$Z$} & \multicolumn{1}{l}{$t$} & \multicolumn{1}{c}{ BA } & \multicolumn{1}{c}{ Voxels } & Brain area \\
\hline 9 & 39 & 24 & 4.85 & 9 & & Rostral Anterior Cingulate Cortex \\
24 & 42 & 30 & 4.1 & 10 & 319 & R Superior Frontal Gyrus \\
27 & 39 & 39 & 3.92 & 9 & & R Medial Frontal Gyrus \\
\hline-24 & 39 & 27 & 4.06 & 9 & 78 & L Medial Frontal Gyrus \\
-12 & 30 & 27 & 3.19 & 32 & & L Superior Frontal Gyrus \\
\hline
\end{tabular}


Figure 1. Schema of experimental design. The English version of the Go/No-Go task was used for Caucasian Americans and Japanese Americans, while the Japanese version was used for Native Japanese. In both versions, participants were told to press a button to every letter shown ("target"), excepted for one designated letter ("non-target"). The letter “v" and “レ” were nontargets for the English and Japanese versions, respectively. There were two Block conditions: the Go Block, where 20 targets were presented, and the Go/No-Go Block, where 10 targets and 10 nontargets were presented. Each of 20 letters in each block was presented for $500 \mathrm{~ms}$, followed by an inter-stimulus interval of $1 \mathrm{~s}$, giving rise to a 30 -s block. Blocks were separated by 20 -s rest periods, where a fixation cross was shown. The order of blocks was pseudo-randomized as shown at the bottom of the figure. The participants had no knowledge of this order of blocks.
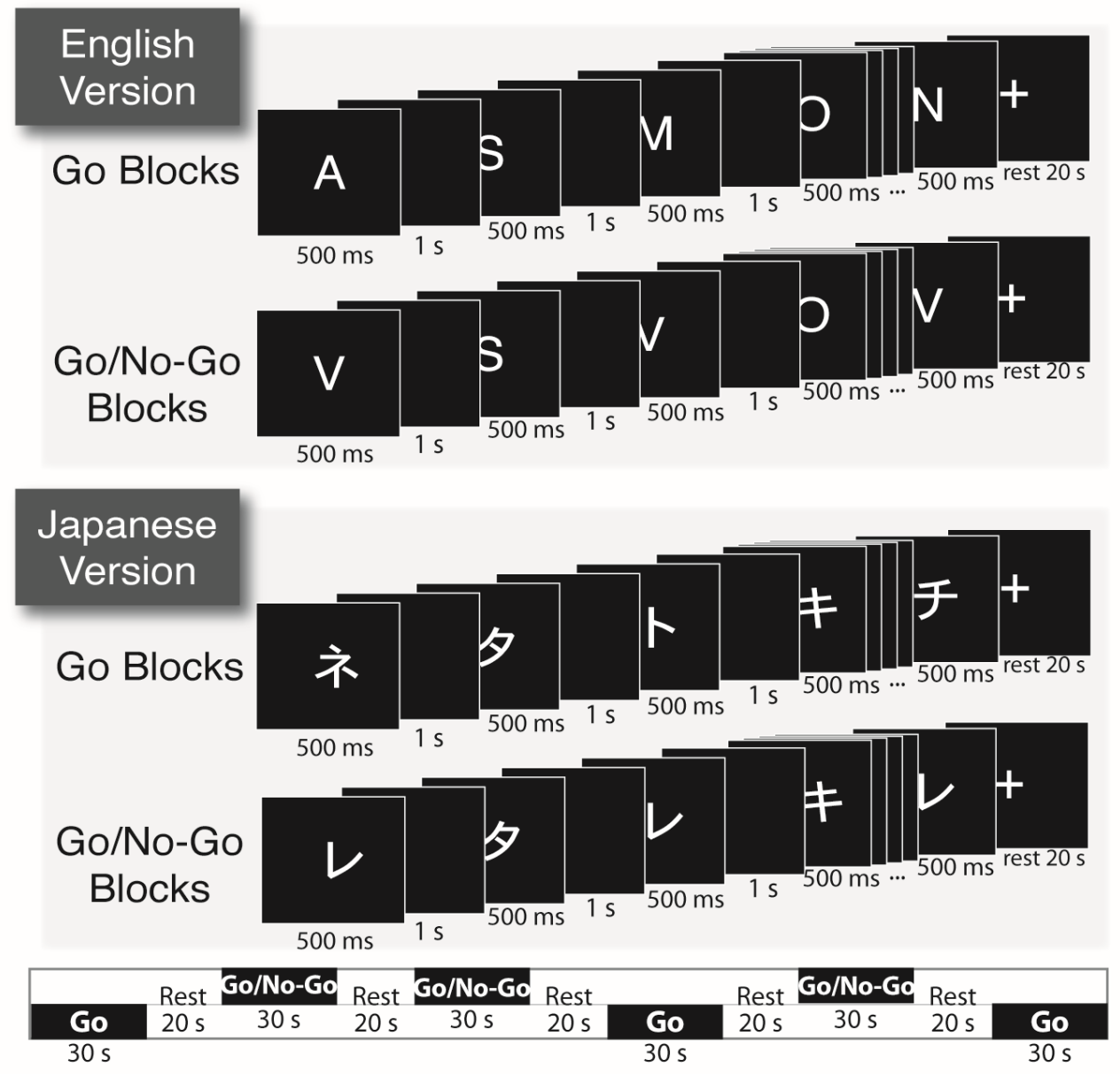
Figure 2. Neural correlates of inhibitory control [Go/No-Go $>$ Go] in the L-IFG as a function of cultural groups. (a) Native Japanese participants had stronger L-IFG activity [peak at $-39,24,3$ ] (that surpassed 3dClustSim-corrected cluster extent threshold, $\mathrm{p}<.005$ clusterwise corrected at $p$ $=.05$ ) than Caucasian Americans and Japanese Americans [Native Japanese $>$ (Caucasian Americans \& Japanese Americans)]. The color scale (red-yellow) indicates t-value. (b) ROI analysis within the L-IFG as defined from a peak voxel in Brown et al. (2006) [-32, 25,-1]. Error bars represent one standard error around the means. CA, JA and JP denote Caucasian Americans, Japanese Americans, and Native Japanese respectively.

a
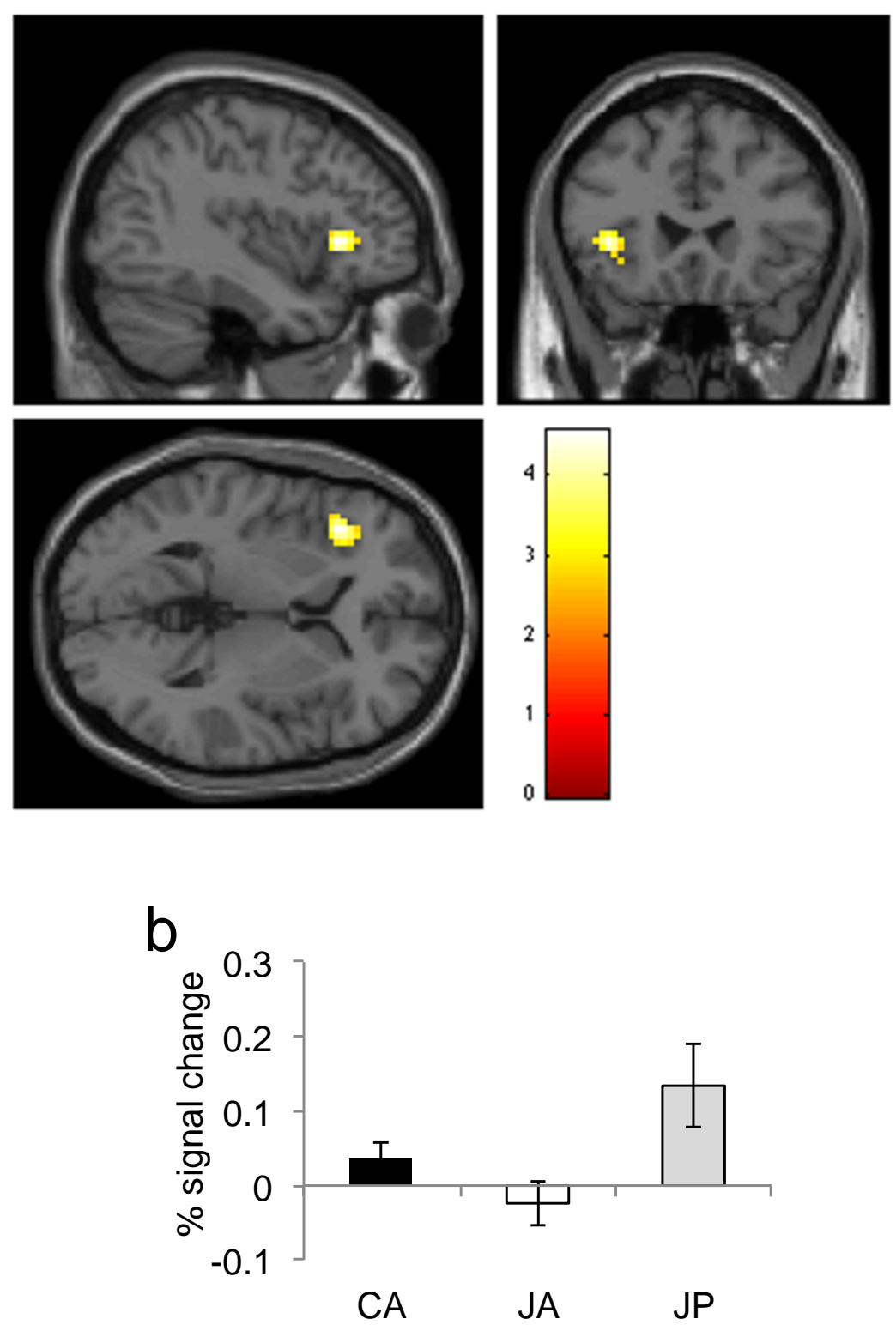
Figure 3. Neural correlates of inhibitory control $[\mathrm{Go} / \mathrm{No}-\mathrm{Go}>\mathrm{Go}]$ in the $\mathrm{rACC}$ as a function of behavioral consistency and behavioral performance. (a) Regression analysis of the positive relationship with behavioral consistency showing increased activity (that surpassed 3dClustSimcorrected cluster extent threshold, $\mathrm{p}<.005$ clusterwise corrected at $p=.05$ ) in the rACC [peak at 636 12] among people with high behavioral consistency. The color scale (red-yellow) indicates tvalue. (b) Scatterplot showing correlation between rACC activity, defined by [Go/No-Go $>$ Go] contrast collapsed across all participants $[-3,33,15]$, and behavioral consistency $(r(56)=.44, p=$ $\left..001 ; r_{\mathrm{s}}(56)=.48, p=.001\right)$. (c) Scatterplot showing correlation between rACC activity and RT difference score [RT in Go/No-Go - Go blocks] $\left(r(56)=.27, \mathrm{p}=.04 ; r_{\mathrm{s}}(56)=.26, p=.05\right)$. (d) Scatterplot showing correlation between rACC activity and Accuracy Rate difference score [Accuracy Rate Difference in Go/No-Go - Go blocks] $(r(56)=-.35, p=.007 ; r \mathrm{~s}(56)=-.24, p=$ .07). CA, JA and JP denote Caucasian Americans, Japanese Americans and Native Japanese respectively.
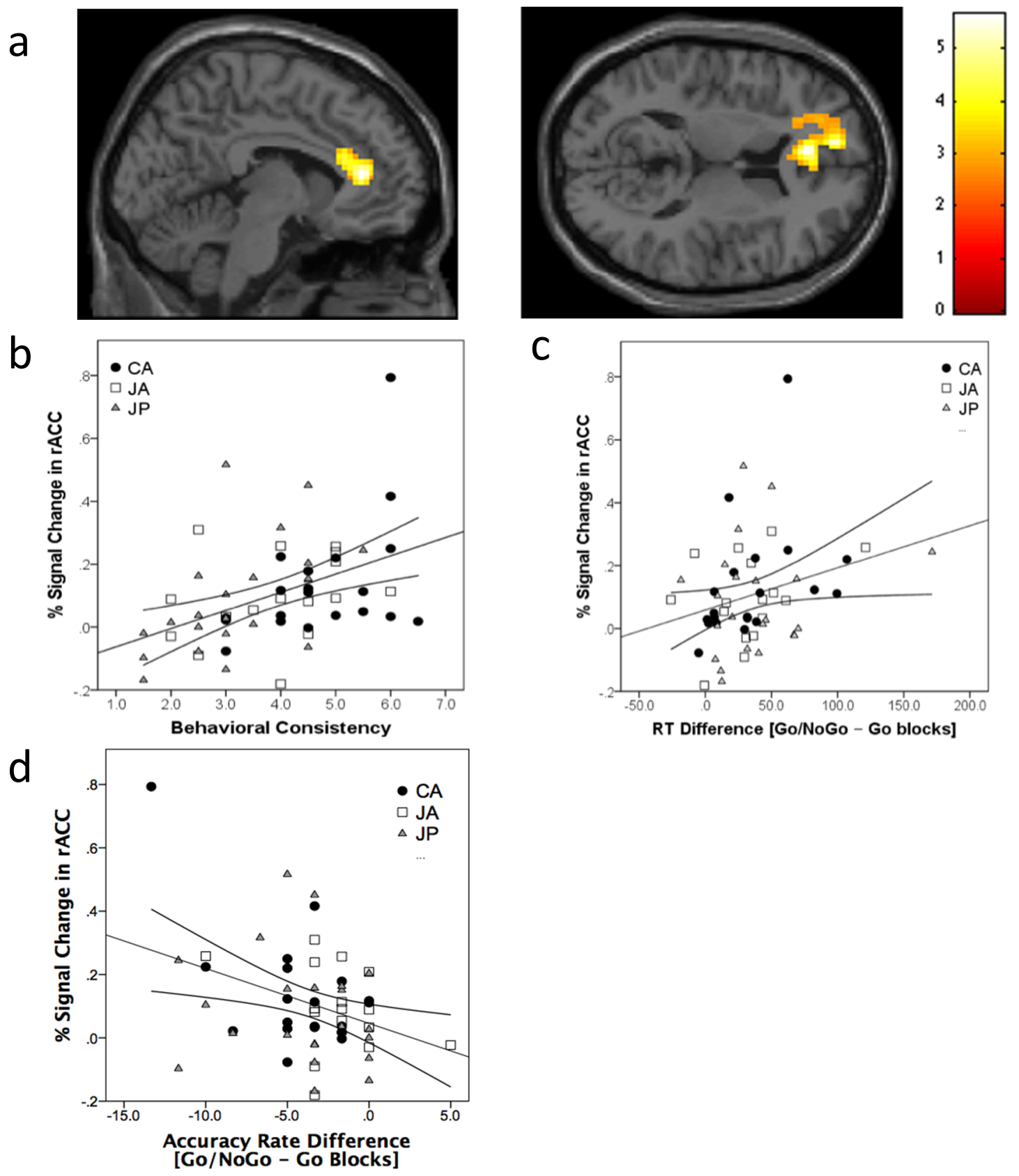
Figure 4. Neural regions [at $\mathrm{x}=9, \mathrm{y}=39, \mathrm{z}=20$ ] showing stronger functional connectivity with the L-IFG seed [-32, 25,-1] (Brown et al., 2006) during the Go/No-Go blocks, compared to the Go blocks, on the whole sample that surpassed 3dClustSim-corrected cluster extent threshold $(p<.005$ clusterwise corrected at $p=.05)$. Overall, participants across groups had stronger functional connectivity between the L-IFG and rACC/medial frontal gyri [peak at 9, 39, 20]. The color scale (red-yellow) indicates t-value.
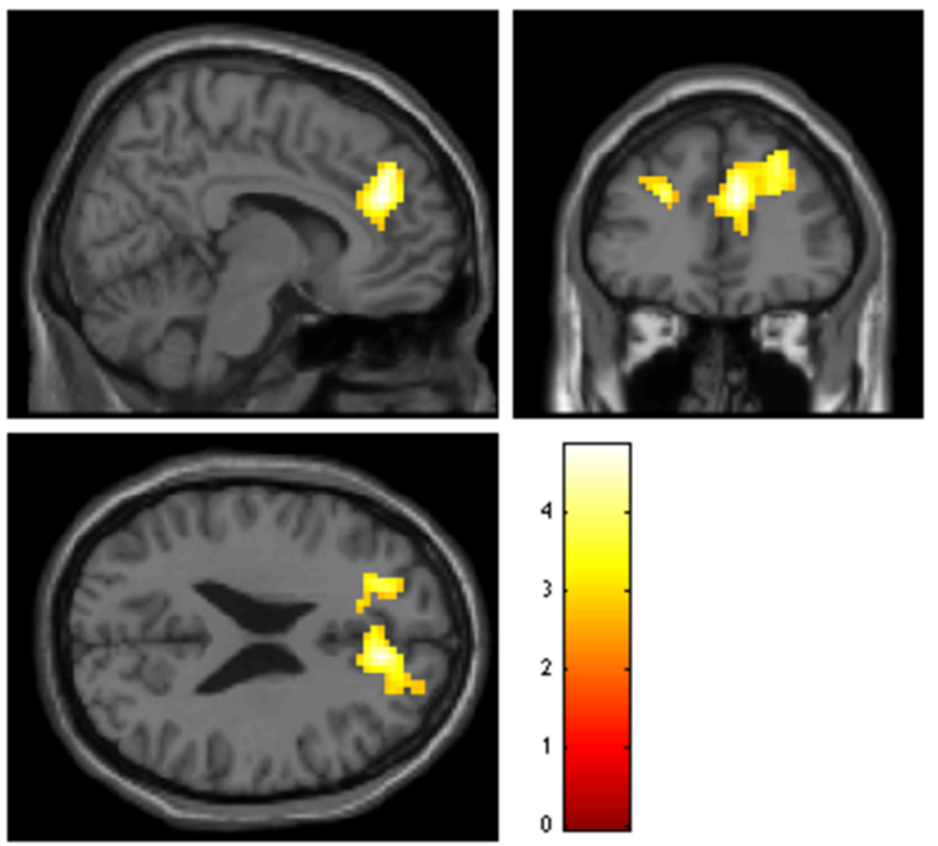
Running Head: CULTURE AND NEURAL BASIS OF INHIBITORY CONTROL

\section{References}

Bogacz, R., Wagenmakers, E.-J., Forstmann, B. U., \& Nieuwenhuis, S. (2010). The neural basis of the speed-accuracy tradeoff. Trends in Neurosciences, 33(1), 10-16. doi:http://dx.doi.org/10.1016/j.tins.2009.09.002

Braver, T. S., Barch, D. M., Gray, J. R., Molfese, D. L., \& Snyder, A. (2001). Anterior cingulate cortex and response conflict: Effects of frequency, inhibition and errors. Cerebral Cortex, 11(9), 825-836. doi:10.1093/cercor/11.9.825

Brown, S. M., Manuck, S. B., Flory, J. D., \& Hariri, A. R. (2006). Neural basis of individual differences in impulsivity: Contributions of corticolimbic circuits for behavioral arousal and control. Emotion, 6(2), 239-245. doi:10.1037/1528-3542.6.2.239

Buchsbaum, B. R., Greer, S., Chang, W.-L., \& Berman, K. F. (2005). Meta-Analysis of Neuroimaging Studies of the Wisconsin Card-Sorting Task and Component Processes. Human Brain Mapping, 25(1), 35-45. doi:10.1002/hbm.20128

Carver, C. S., \& White, T. L. (1994). Behavioral inhibition, behavioral activation, and affective responses to impending reward and punishment: The BIS/BAS Scales. Journal of Personality and Social Psychology, 67(2), 319-333. doi:10.1037/00223514.67.2.319

Chao, R., \& Tseng, V. (2002). Parenting of Asians. In M. H. Bornstein (Ed.), Handbook of parenting: Vol. 4: Social conditions and applied parenting (2nd ed., pp. 59-93). Mahwah, NJ: Lawrence Erlbaum Associates Publishers.

Cheon, B. K., Im, D.-M., Harada, T., Kim, J.-S., Mathur, V. A., Scimeca, J. M., . . Chiao, J. Y. (2013). Cultural modulation of the neural correlates of emotional pain perception: The role of other-focusedness. Neuropsychologia, 51(7), 1177-1186. doi:http://dx.doi.org/10.1016/j.neuropsychologia.2013.03.018

Chiao, J. Y., \& Blizinsky, K. D. (2010). Culture-gene coevolution of individualismcollectivism and the serotonin transporter gene. Proceedings of the Royal Society B: Biological Sciences, 277(1681), 529-537. doi:rspb.2009.1650 [pii]

10.1098/rspb.2009.1650

Chiao, J. Y., Cheon, B. K., Pornpattananangkul, N., Mrazek, A. J., \& Blizinsky, K. D. (2013). Cultural Neuroscience: Progress and Promise. Psychological Inquiry, 24(1), 1-19. doi:10.1080/1047840X.2013.752715

Chiao, J. Y., Harada, T., Komeda, H., Li, Z., Mano, Y., Saito, D., . . Iidaka, T. (2009a). Dynamic Cultural Influences on Neural Representations of the Self. Journal of Cognitive Neuroscience, 22(1), 1-11. doi:10.1162/jocn.2009.21192 
Running Head: CULTURE AND NEURAL BASIS OF INHIBITORY CONTROL

Chiao, J. Y., Harada, T., Komeda, H., Li, Z., Mano, Y., Saito, D., . . Iidaka, T. (2009b). Neural basis of individualistic and collectivistic views of self. Human Brain Mapping, 30(9), 2813-2820. doi:10.1002/hbm.20707

Chiao, J. Y., Hariri, A. R., Harada, T., Mano, Y., Sadato, N., Parrish, T. B., \& Iidaka, T. (2010). Theory and methods in cultural neuroscience. Social Cognitive and Affective Neuroscience, 5(2-3), 356-361. doi:10.1093/scan/nsq063

Chikamatsu, N., Yokoyama, S., Nozaki, H., Long, E., \& Fukuda, S. (2000). A Japanese logographic character frequency list for cognitive science research. Behavior Research Methods, Instruments, \& Computers, 32(3), 482-500. doi:10.3758/bf03200819

Chikazoe, J., Konishi, S., Asari, T., Jimura, K., \& Miyashita, Y. (2007). Activation of right inferior frontal gyrus during response inhibition across response modalities. Journal of Cognitive Neuroscience, 19(1), 69-80. doi:10.1162/jocn.2007.19.1.69

Chuah, Y. M. L., Venkatraman, V., Dinges, D. F., \& Chee, M. W. L. (2006). The Neural Basis of Interindividual Variability in Inhibitory Efficiency after Sleep Deprivation. The Journal of Neuroscience, 26(27), 7156-7162. doi:10.1523/jneurosci.090606.2006

Coffey, D. M., Marmol, L., Schock, L., \& Adams, W. (2005). The influence of acculturation on the Wisconsin Card Sorting Test by Mexican Americans. Archives of Clinical Neuropsychology, 20(6), 795-803. doi:10.1016/j.acn.2005.04.009

Cortina, J. M. (1993). What is coefficient alpha? An examination of theory and applications. Journal of Applied Psychology, 78(1), 98-104. doi:10.1037/0021-9010.78.1.98

Cox, R. W. (1996). AFNI: Software for Analysis and Visualization of Functional Magnetic Resonance Neuroimages. Computers and Biomedical Research, 29(3), 162-173. doi:http://dx.doi.org/10.1006/cbmr.1996.0014

Criaud, M., \& Boulinguez, P. (2013). Have we been asking the right questions when assessing response inhibition in go/no-go tasks with fMRI? A meta-analysis and critical review. Neuroscience \& Biobehavioral Reviews, 37(1), 11-23. doi:S01497634(12)00193-5 [pii]

10.1016/j.neubiorev.2012.11.003

Cross, S. E., Hardin, E. E., \& Gercek-Swing, B. (2011). The What, How, Why, and Where of Self-Construal. Personality and Social Psychology Review, 15(2), 142-179. doi: $10.1177 / 1088868310373752$

de Greck, M., Shi, Z., Wang, G., Zuo, X., Yang, X., Wang, X., .. . Han, S. (2012). Culture modulates brain activity during empathy with anger. Neuroimage, 59(3), 28712882. doi:http://dx.doi.org/10.1016/j.neuroimage.2011.09.052 
Running Head: CULTURE AND NEURAL BASIS OF INHIBITORY CONTROL

de Zubicaray, G., Andrew, C., Zelaya, F., Williams, S., \& Dumanoir, C. (2000). Motor response suppression and the prepotent tendency to respond: a parametric fMRI study. Neuropsychologia, 38(9), 1280 - 1291.

Dekker, S., \& Fischer, R. (2008). Cultural Differences in Academic Motivation Goals: A Meta-Analysis Across 13 Societies. The Journal of Educational Research, 102(2), 99-110. doi:10.3200/JOER.102.2.99-110

Dosenbach, N. U., Visscher, K. M., Palmer, E. D., Miezin, F. M., Wenger, K. K., Kang, H. C., . . . Petersen, S. E. (2006). A core system for the implementation of task sets. Neuron, 50(5), 799-812. doi:S0896-6273(06)00349-7 [pii]

10.1016/j.neuron.2006.04.031

Ekman, P. (1971). Universals and cultural differences in facial expressions of emotion. Nebraska Symposium on Motivation, 19, 207-283.

Fassbender, C., Murphy, K., Foxe, J. J., Wylie, G. R., Javitt, D. C., Robertson, I. H., \& Garavan, H. (2004). A topography of executive functions and their interactions revealed by functional magnetic resonance imaging. Cognitive Brain Research, 20(2), 132-143. doi:http://dx.doi.org/10.1016/j.cogbrainres.2004.02.007

Friedman, L., \& Glover, G. H. (2006). Reducing interscanner variability of activation in a multicenter fMRI study: controlling for signal-to-fluctuation-noise-ratio (SFNR) differences. Neuroimage, 33(2), 471-481. doi:S1053-8119(06)00794-4 [pii]

10.1016/j.neuroimage.2006.07.012

Friston, K. J., Buechel, C., Fink, G. R., Morris, J., Rolls, E., \& Dolan, R. J. (1997). Psychophysiological and Modulatory Interactions in Neuroimaging. Neuroimage, 6(3), 218-229. doi:http://dx.doi.org/10.1006/nimg.1997.0291

Friston, K. J., Holmes, A. P., Worsley, K. J., Poline, J. P., Frith, C. D., \& Frackowiak, R. S. J. (1994). Statistical parametric maps in functional imaging: A general linear approach. Human Brain Mapping, 2(4), 189-210. doi:10.1002/hbm.460020402

Funder, D. C., \& Colvin, C. R. (1991). Explorations in behavioral consistency: Properties of persons, situations, and behaviors. Journal of Personality and Social Psychology, 60(5), 773-794. doi:10.1037/0022-3514.60.5.773

Gagne, J. R., \& Saudino, K. J. (2010). Wait For It! A Twin Study of Inhibitory Control in Early Childhood. Behavior Genetics, 40(3), 327-337. doi:10.1007/s10519-0099316-6

Gagne, J. R., Saudino, K. J., \& Asherson, P. (2011). The genetic etiology of inhibitory control and behavior problems at 24 months of age. Journal of Child Psychology \& Psychiatry, 52(11), 1155-1163. doi:10.1111/j.1469-7610.2011.02420.x 
Running Head: CULTURE AND NEURAL BASIS OF INHIBITORY CONTROL

Garavan, H., Ross, T. J., \& Stein, E. A. (1999). Right hemispheric dominance of inhibitory control: An event-related functional MRI study. Proceedings of the National Academy of Sciences, 96(14), 8301-8306. doi:10.1073/pnas.96.14.8301

Gitelman, D. R., Penny, W. D., Ashburner, J., \& Friston, K. J. (2003). Modeling regional and psychophysiologic interactions in fMRI: the importance of hemodynamic deconvolution. Neuroimage, 19(1), 200-207. doi:http://dx.doi.org/10.1016/S1053$\underline{8119(03) 00058-2}$

Grant, D. A., \& Berg, E. (1948). A behavioral analysis of degree of reinforcement and ease of shifting to new responses in a Weigl-type card-sorting problem. Journal of Experimental Psychology, 38(4), 404-411. doi:10.1037/h0059831

Han, S., Northoff, G., Vogeley, K., Wexler, B. E., Kitayama, S., \& Varnum, M. E. W. (2013). A Cultural Neuroscience Approach to the Biosocial Nature of the Human Brain. Annual Review of Psychology, 64(1), 335-359. doi:doi:10.1146/annurevpsych-071112-054629

Hardin, E. E. (2006). Convergent evidence for the multidimensionality of self-construal. Journal of Cross-Cultural Psychology, 37(5), 516-521. doi:Doi $10.1177 / 0022022106290475$

Hardin, E. E., Leong, F. T. L., \& Bhagwat, A. A. (2004). Factor structure of the selfconstrual scale revisited - Implications for the multidimensionality of self-construal. Journal of Cross-Cultural Psychology, 35(3), 327-345. doi:Doi $10.1177 / 0022022104264125$

Hariri, A. R., Tessitore, A., Mattay, V. S., Fera, F., \& Weinberger, D. R. (2002). The Amygdala Response to Emotional Stimuli: A Comparison of Faces and Scenes. Neuroimage, 17(1), 317-323. doi:http://dx.doi.org/10.1006/nimg.2002.1179

Hirose, S., Chikazoe, J., Watanabe, T., Jimura, K., Kunimatsu, A., Abe, O., . . Konishi, S. (2012). Efficiency of Go/No-Go Task Performance Implemented in the Left Hemisphere. The Journal of Neuroscience, 32(26), 9059-9065. doi:10.1523/jneurosci.0540-12.2012

Horn, N. R., Dolan, M., Elliott, R., Deakin, J. F. W., \& Woodruff, P. W. R. (2003). Response inhibition and impulsivity: An fMRI study. Neuropsychologia, 41(14), 1959-1966. doi:10.1016/s0028-3932(03)00077-0

Kanagawa, C., Cross, S. E., \& Markus, H. R. (2001). 'Who am I?' The cultural psychology of the conceptual self. Personality and Social Psychology Bulletin, 27(1), 90-103. doi:10.1177/0146167201271008 
Running Head: CULTURE AND NEURAL BASIS OF INHIBITORY CONTROL

Kiehl, K. A., Liddle, P. F., \& Hopfinger, J. B. (2000). Error processing and the rostral anterior cingulate: An event-related fMRI study. Psychophysiology, 37(2), 216-223. doi:10.1017/s0048577200990231

Kitayama, S., Park, H., Sevincer, A. T., Karasawa, M., \& Uskul, A. K. (2009). A cultural task analysis of implicit independence: Comparing North America, Western Europe, and East Asia. Journal of Personality and Social Psychology, 97(2), 236-255. doi:10.1037/a0015999

Kwon, Y.-I. (2002). Western influences in Korean preschool education. International Education Journal, 3(3), 153-164.

Lahat, A., Todd, R., Mahy, C. E. V., Lau, K., \& Zelazo, P. D. (2010). Neurophysiological correlates of executive function: a comparison of european-canadian and chinesecanadian 5-year-olds. Frontiers in Human Neuroscience, 3. doi:10.3389/neuro.09.072.2009

Langenecker, S. A., Kennedy, S. E., Guidotti, L. M., Briceno, E. M., Own, L. S., Hooven, T., ... Zubieta, J.-K. (2007). Frontal and limbic activation during inhibitory control predicts treatment response in major depressive disorder. Biological Psychiatry, 62(11), 1272-1280. doi:10.1016/j.biopsych.2007.02.019

Levene, H. (1960). Robust tests for equality of variances1. Contributions to probability and statistics: Essays in honor of Harold Hotelling, 2, 278-292.

Levine, T. R., Bresnahan, M. J., Park, H. S., Lapinsky, M. K., Wittenbaum, G. M., Shearman, S. M., . . Ohashi, R. (2003). Self-construal scales lack validity. Human Communication Research, 29(2), 210-252. doi:10.1111/j.14682958.2003.tb00837.x

Lewand, R. (2000). Cryptological mathematics: MAA.

Ma, Y., Bang, D., Wang, C., Allen, M., Frith, C., Roepstorff, A., \& Han, S. (2014). Sociocultural patterning of neural activity during self-reflection. Social Cognitive and Affective Neuroscience, 9(1), 73-80. doi:10.1093/scan/nss103

Maltby, N., Tolin, D. F., Worhunsky, P., O'Keefe, T. M., \& Kiehl, K. A. (2005). Dysfunctional action monitoring hyperactivates frontal-striatal circuits in obsessive-compulsive disorder: an event-related fMRI study. Neuroimage, 24(2), 495-503. doi:10.1016/j.neuroimage.2004.08.041

Markus, H. R., \& Kitayama, S. (1991). Culture and the self: Implications for cognition, emotion, and motivation. Psychological Review, 98(2), 224-253. doi:10.1037/0033-295x.98.2.224 
Running Head: CULTURE AND NEURAL BASIS OF INHIBITORY CONTROL

Matsumoto, D. (1999). Culture and self: An empirical assessment of Markus and Kitayama's theory of independent and interdependednt self-construal. Asian Journal of Social Psychology, 2(3), 289-310. doi:10.1111/1467-839x.00042

Matsumoto, D., Yoo, S. H., Hirayama, S., \& Petrova, G. (2005). Development and Validation of a Measure of Display Rule Knowledge: The Display Rule Assessment Inventory. Emotion, 5(1), 23-40. doi:10.1037/1528-3542.5.1.23

Mazzola-Pomietto, P., Kaladjian, A., Azorin, J.-M., Anton, J.-L., \& Jeanningros, R. (2009). Bilateral decrease in ventrolateral prefrontal cortex activation during motor response inhibition in mania. Journal of Psychiatric Research, 43(4), 432-441. doi:10.1016/j.jpsychires.2008.05.004

McLaren, D. G., Ries, M. L., Xu, G., \& Johnson, S. C. (2012). A generalized form of context-dependent psychophysiological interactions (gPPI): A comparison to standard approaches. Neuroimage, 61(4), 1277-1286. doi:http://dx.doi.org/10.1016/j.neuroimage.2012.03.068

Menon, V., Adleman, N. E., White, C. D., Glover, G. H., \& Reiss, A. L. (2001). Errorrelated brain activation during a $\mathrm{Go} / \mathrm{NoGo}$ response inhibition task. Human Brain Mapping, 12(3), 131-143. doi:10.1002/1097-0193(200103)12:3<131::aidhbm1010>3.0.co;2-c

Mrazek, A., Chiao, J., Blizinsky, K., Lun, J., \& Gelfand, M. (2013). The role of culturegene coevolution in morality judgment: examining the interplay between tightnesslooseness and allelic variation of the serotonin transporter gene. Culture and Brain, 1(2-4), 100-117. doi:10.1007/s40167-013-0009-x

Na, J., Grossmann, I., Varnum, M. E. W., Kitayama, S., Gonzalez, R., \& Nisbett, R. E. (2010). Cultural differences are not always reducible to individual differences. Proceedings of the National Academy of Sciences, 107(14), 6192-6197. doi:10.1073/pnas.1001911107

Nakata, H., Inui, K., Wasaka, T., Akatsuka, K., \& Kakigi, R. (2005). Somato-motor inhibitory processing in humans: A study with MEG and ERP. European Journal of Neuroscience, 22(7), 1784-1792. doi:10.1111/j.1460-9568.2005.04368.x

Nieuwenhuis, S., Yeung, N., Van Den Wildenberg, W., \& Ridderinkhof, K. R. (2003). Electrophysiological correlates of anterior cingulate function in a go/no-go task: Effects of response conflict and trial type frequency. Cognitive, Affective \& Behavioral Neuroscience, 3(1), 17-26. doi:10.3758/cabn.3.1.17

Noguchi, K. (2007). Examination of the content of individualism/collectivism scales in cultural comparisons of the USA and Japan. Asian Journal of Social Psychology, 10(3), 131-144. doi:10.1111/j.1467-839X.2007.00220.x 
Running Head: CULTURE AND NEURAL BASIS OF INHIBITORY CONTROL

Nunnally, J. C. (1978). Psychometric theory (2d ed.). New York: : McGraw-Hill.

O’Reilly, J. X., Woolrich, M. W., Behrens, T. E. J., Smith, S. M., \& Johansen-Berg, H. (2012). Tools of the trade: psychophysiological interactions and functional connectivity. Social Cognitive and Affective Neuroscience, 7(5), 604-609. doi:10.1093/scan/nss055

Oh, S., \& Lewis, C. (2008). Korean preschoolers' advanced inhibitory control and its relation to other executive skills and mental state understanding. Child Development, 79(1), 80-99. doi:10.1111/j.1467-8624.2007.01112.x

Oyserman, D., Coon, H. M., \& Kemmelmeier, M. (2002). Rethinking individualism and collectivism: Evaluation of theoretical assumptions and meta-analyses. Psychological Bulletin, 128(1), 3-72. doi:10.1037/0033-2909.128.1.3

Oyserman, D., \& Uskul, A. K. (2008). Individualism and collectivism: Societal-level processes with implications for individual-level and society-level outcomes. In F. J. R. van de Vijver, D. A. van Hemert, \& Y. H. Poortinga (Eds.), Multilevel analysis of individuals and cultures. (pp. 145-173, xii, 448). New York, NY: Taylor \& Francis Group/Lawrence Erlbaum Associates.

Parrish, T. B., Gitelman, D. R., LaBar, K. S., \& Mesulam, M. M. (2000). Impact of signalto-noise on functional MRI. Magn Reson Med, 44(6), 925-932. doi:10.1002/15222594(200012)44:6<925::AID-MRM14>3.0.CO;2-M [pii]

Perri, R. L., Berchicci, M., Spinelli, D., \& Di Russo, F. (2014). Individual Differences in Response Speed and Accuracy are Associated to Specific Brain Activities of two Interacting Systems. Frontiers in Behavioral Neuroscience, 8. doi:10.3389/fnbeh.2014.00251

Razani, J., Burciaga, J., Madore, M., \& Wong, J. (2007). Effects of acculturation on tests of attention and information processing in an ethnically diverse group. Arch Clin Neuropsychol, 22(3), 333-341. doi:S0887-6177(07)00015-7 [pii]

10.1016/j.acn.2007.01.008

Sabbagh, M. A., Xu, F., Carlson, S. M., Moses, L. J., \& Lee, K. (2006). The Development of Executive Functioning and Theory of Mind: A Comparison of Chinese and U.S. Preschoolers. Psychological Science, 17(1), 74-81. doi:10.1111/j.14679280.2005.01667.x

Siegel, S. (1956). Nonparametric statistics for the behavioral sciences. New York, NY, US: McGraw-Hill.

Simmonds, D. J., Pekar, J. J., \& Mostofsky, S. H. (2008). Meta-analysis of Go/No-go tasks demonstrating that fMRI activation associated with response inhibition is task- 
Running Head: CULTURE AND NEURAL BASIS OF INHIBITORY CONTROL

dependent. Neuropsychologia, $\quad$ 224-232. doi:10.1016/j.neuropsychologia.2007.07.015

Singelis, T. M. (1994). The Measurement of Independent and Interdependent SelfConstruals. Personality and Social Psychology Bulletin, 20(5), 580-591. doi:Doi 10.1177/0146167294205014

Smith, J. L., Johnstone, S. J., \& Barry, R. J. (2008). Movement-related potentials in the Go/NoGo task: The P3 reflects both cognitive and motor inhibition. Clinical Neurophysiology, $119(3)$, 704-714. doi:http://dx.doi.org/10.1016/j.clinph.2007.11.042

Somech, A. (2000). The independent and the interdependent selves: Different meanings in different cultures. International Journal of Intercultural Relations, 24(2), 161-172. doi:10.1016/s0147-1767(99)00030-9

Steele, V. R., Aharoni, E., Munro, G. E., Calhoun, V. D., Nyalakanti, P., Stevens, M. C., .. . Kiehl, K. A. (2013). A large scale $(\mathrm{N}=102)$ functional neuroimaging study of response inhibition in a Go/NoGo task. Behavioural Brain Research, 256 256, 529536. doi:10.1016/j.bbr.2013.06.001

Suh, E. M. (2002). Culture, identity consistency, and subjective well-being. Journal of Personality and Social Psychology, 83(6), 1378-1391. doi:10.1037/00223514.83.6.1378

Sul, S., Choi, I., \& Kang, P. (2011). Cultural modulation of self-referential brain activity for personality traits and social identities. Social Neuroscience, 7(3), 280-291. doi:10.1080/17470919.2011.614001

Sutton, B. P., Goh, J., Hebrank, A., Welsh, R. C., Chee, M. W., \& Park, D. C. (2008). Investigation and validation of intersite fMRI studies using the same imaging hardware. Journal of Magnetic Resonance Imaging, 28(1), 21-28. doi:10.1002/jmri.21419

Swick, D., Ashley, V., \& Turken, A. (2008). Left inferior frontal gyrus is critical for response inhibition. BMC Neuroscience, 9(1), 102.

Swick, D., Ashley, V., \& Turken, U. (2011). Are the neural correlates of stopping and not going identical? Quantitative meta-analysis of two response inhibition tasks. Neuroimage, 56(3), 1655-1665. doi:10.1016/j.neuroimage.2011.02.070

Talairach, J., \& Tournoux, P. (1988). Co-planar stereotaxic atlas of the human brain (Vol. 147): Thieme New York: 
Running Head: CULTURE AND NEURAL BASIS OF INHIBITORY CONTROL

Tamm, L., Menon, V., \& Reiss, A. L. (2002). Maturation of brain function associated with response inhibition. Journal of the American Academy of Child \& Adolescent Psychiatry, 41(10), 1231-1238. doi:10.1097/00004583-200210000-00013

Tanaka, A., \& Yamauchi, H. (2004). Cultural self-construal and achievement goal. Hellenic Journal of Psychology, 1, 221-237.

Urdan, T. (2004). Predictors of Academic Self-Handicapping and Achievement: Examining Achievement Goals, Classroom Goal Structures, and Culture. Journal of Educational Psychology, 96(2), 251-264. doi:10.1037/0022-0663.96.2.251

van de Vijver, F. J. R., van Hemert, D. A., \& Poortinga, Y. H. (2008). Conceptual issues in multilevel models. In F. J. R. van de Vijver, D. A. van Hemert, \& Y. H. Poortinga (Eds.), Multilevel analysis of individuals and cultures. (pp. 3-26, xii, 448). New York, NY: Taylor \& Francis Group/Lawrence Erlbaum Associates.

Wang, J., \& Mao, S. (1996). Culture and the kindergarten curriculum in the People's Republic of China. Early Child Development and Care, 123, 143-156. doi:10.1080/0300443961230110

Watanabe, J., Sugiura, M., Sato, K., Sato, Y., Maeda, Y., Matsue, Y., . . Kawashima, R. (2002). The Human Prefrontal and Parietal Association Cortices Are Involved in NO-GO Performances: An Event-Related fMRI Study. Neuroimage, 17(3), 12071216. doi:http://dx.doi.org/10.1006/nimg.2002.1198

Zheng, D., Oka, T., Bokura, H., \& Yamaguchi, S. (2008). The key locus of common response inhibition network for no-go and stop signals. Journal of Cognitive Neuroscience, 20(8), 1434-1442. doi:10.1162/jocn.2008.20100 